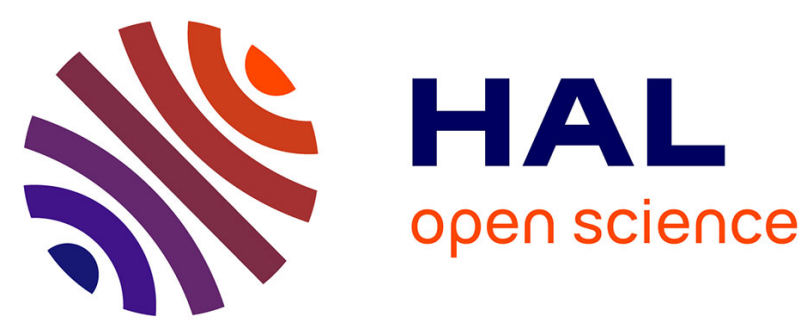

\title{
Acoustic and Large Eddy Simulation studies of azimuthal modes in annular combustion chambers
}

Pierre Wolf, Gabriel Staffelbach, Laurent Y.M. Gicquel, Jens-Dominik Müller, Thierry Poinsot

\section{- To cite this version:}

Pierre Wolf, Gabriel Staffelbach, Laurent Y.M. Gicquel, Jens-Dominik Müller, Thierry Poinsot. Acoustic and Large Eddy Simulation studies of azimuthal modes in annular combustion chambers. Combustion and Flame, 2012, vol. 159, pp.3398-3413. 10.1016/j.combustflame.2012.06.016 . hal-00801825

\section{HAL Id: hal-00801825 \\ https://hal.science/hal-00801825}

Submitted on 18 Mar 2013

HAL is a multi-disciplinary open access archive for the deposit and dissemination of scientific research documents, whether they are published or not. The documents may come from teaching and research institutions in France or abroad, or from public or private research centers.
L'archive ouverte pluridisciplinaire HAL, est destinée au dépôt et à la diffusion de documents scientifiques de niveau recherche, publiés ou non, émanant des établissements d'enseignement et de recherche français ou étrangers, des laboratoires publics ou privés. 


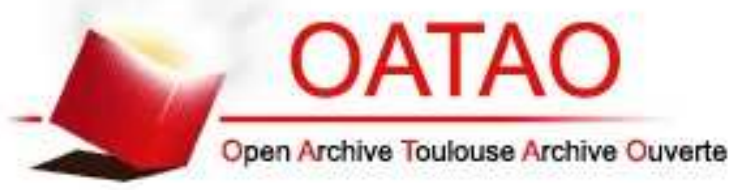

\section{Open Archive TOULOUSE Archive Ouverte (OATAO)}

OATAO is an open access repository that collects the work of Toulouse researchers and makes it freely available over the web where possible.

This is an author-deposited version published in : http://oatao.univ-toulouse.fr/ Eprints ID : 8579

To link to this article : DOI:10.1016/j.combustflame.2012.06.016

URL : http://dx.doi.org/10.1016/j.combustflame.2012.06.016

To cite this version :

Wolf, Pierre and Staffelbach, Gabriel and Gicquel, Laurent Y.M. and Müller, Jens-Dominik and Poinsot, Thierry Acoustic and Large Eddy Simulation studies of azimuthal modes in annular combustion chambers. (2012) Combustion and Flame, vol. $159\left(\mathrm{n}^{\circ}\right.$ 11). pp. 3398-3413. ISSN 0010-2180

Any correspondence concerning this service should be sent to the repository administrator: staff-oatao@ listes.diff.inp-toulouse.fr 


\title{
Acoustic and Large Eddy Simulation studies of azimuthal modes in annular combustion chambers.
}

\author{
P. Wolf ${ }^{\mathrm{a}, *}$, G. Staffelbach ${ }^{\mathrm{a}}$, L.Y.M. Gicquel ${ }^{\mathrm{a}}$, J.-D. Müller ${ }^{\mathrm{b}}$, T. Poinsot ${ }^{\mathrm{c}}$ \\ ${ }^{a}$ CERFACS, Toulouse, France \\ ${ }^{b}$ Queen Mary, University of London, England \\ ${ }^{c}$ IMFT, Toulouse, France
}

\begin{abstract}
The objectives of this paper are the description of azimuthal instability modes found in annular combustion chambers using two numerical tools: (1) Large Eddy Simulation (LES) methods and (2) acoustic solvers. These strong combustion instabilities are difficult to study experimentally and the present study is based on a LES of a full aeronautical combustion chamber. The LES exhibits a self-excited oscillation at the frequency of the first azimuthal eigenmode. The mesh independence of the LES is verified before analysing the nature of this mode using various indicators over more than 100 cycles: the mode is mostly a pure standing mode but it transitions from time to time to a turning mode because of turbulent fluctuations, confirming experimental observationsand theoretical results. The correlation between pressure and heat release fluctuations (Rayleigh criterion) is not verified locally but it is satisfied when pressure and heat release are averaged over sectors. LES is also used to check modes predicted by an acoustic Helmholtz solver where the flow is frozen and flames are modelled using Flame Transfer Function (FTF) as done in most present tools. The results in terms of mode structure compare well confirming that the mode appearing in the LES is the first azimuthal mode of the chamber. Moreover, the acoustic solver provides stability maps suggesting that a reduction of the time delay of the FTF would be enough to stabilise the mode. This is confirmed with
\end{abstract}

\footnotetext{
* corresponding author

Email addresses: wolf@cerfacs.fr (P. Wolf), staffelbach@cerfacs.fr (G. Staffelbach)
} 
LES by increasing the flame speed and verifying that this modification leads to a damped mode in a few cycles.

Keywords: Acoustics, Large Eddy Simulation, Azimuthal modes, Annular combustion chambers

\section{Introduction}

Combustion instabilities in annular chambers of gas turbines constitute a major issue for many designs: these powerful instabilities often correspond to azimuthal waves propagating in clockwise and counterclockwise directions in the annular chamber. They lead to vibrations and structural damage $[1,3,4]$. Predicting them at the design stage remains a scientific challenge because many fundamental aspects which control these modes are not yet understood.

Combustion instabilities have been studied for a long time $[5,6,7,8]$ but most academic studies have taken place in simplified configurations which were easier to study numerically and experimentally. The most important simplification to study unstable modes in annular gas turbines is to analyse only one sector of the combustor, avoiding the complexity of a full annular chamber which contains typically 10 to 25 sectors. Obviously, this simplification implies significant assumptions and therefore some care as the physics of instabilities in a single burner and in a full combustor with multiple burners may differ significantly. Knowing how far the single sector method can be applied to real annular chambers is an open question.

Typical acoustic analysis tools (also called Helmholtz solvers) applied to combustion instabilities require the resolution of a wave equation in frequency or time domain. They assume that the flow is frozen and solve the wave equation in a non-isothermal flow with impedance conditions on walls, inlets and outlets to identify eigenmodes $[9,10,11]$. The effect of flames is introduced into acoustic codes through a FTF (Flame Transfer Function) measuring the flame response (quantified by the heat release rate fluctuations $q^{\prime}$ ) to a velocity change at the inlet of the burner $u^{\prime}[12,13,14]$. FTFs are complex-valued depending 
on frequency. A recent extension of these intrinsically linear methods is the introduction of FTFs which depend also on the oscillation amplitude: these FDF (Flame Describing Function) formulations can predict not only the unstable nature of the modes but also their limit cycle amplitudes [7, 15].

Theoretically, the physics of azimuthal modes in annular chambers should not differ significantly from other, simpler configurations: the source of unstable modes in all reacting flows is the well-known flame / acoustics coupling mechanism leading to the various forms of the Rayleigh criterion $[5,10,16]$. Extending the tools used for simpler burners should be a straightforward task for annular chambers. In practice however, the models developed for most academic burners are applicable mainly to longitudinal modes and extending them to the complex modes appearing in real annular chambers is a difficult task. The literature $[3,1,17,18,19,20]$ contains multiple 'extended' methods dedicated to annular chambers which provide the growth rate and the structure of modes (longitudinal or azimuthal). These approaches are usually based on a one-dimensional network-view of the chamber $[9,21,3]$ in which each burner is only influenced by the flow rate fluctuation it is submitted to by the azimuthal acoustic mode, locally, all acoustic waves propagate longitudinally. All burners are supposed to be independent from their neighbours and have the same transfer function, i.e. flame / flame interactions between neighbouring burners are neglected. This assumption has been verified numerically in one annular helicopter chamber [22] but it is not valid in general. In liquid-fueled rocket engines or more generally in burners containing multiple jets [23], the interaction between neighbouring flames can lead to instability and transverse modes. This may also happen in gas turbines and hence invalidate the one-dimensional network approach.

Even for modes where burners can be assumed to respond in a one-dimensional manner to acoustic perturbations, determining their FTFs remains difficult $[24,25,26]$. FTFs are the key elements of acoustic solvers for combustion stability as these solvers require the flame response to the acoustic field as an input data $[11,10]$ and the FTF is the most common solution. FTFs depend on multiple parameters (regime but also pulsation amplitude, wall temperature, 


\begin{tabular}{ccc}
\hline Type & Modes & Description \\
\hline 1 & Standing & Pressure nodes are fixed \\
2 & Turning or spinning & Pressure structure is turning at the sound speed \\
3 & Rotating & Standing mode where the structure slowly rotates \\
& & at the azimuthal convective speed \\
\hline
\end{tabular}

Table 1: A classification of azimuthal modes.

pilot flames, etc) so that an accurate description of FTF is often not available $[27,28,7]$. A second difficulty is to take into account the complexity of the geometry $[29,30]$ which leads to a very large number of possible acoustic modes. Finally, another difficulty is due to the structure of the azimuthal modes [31]: while most academic, single sector experiments exhibit longitudinal modes, in real annular combustion chambers, the first (and sometimes second) azimuthal acoustic mode is often the strongest mode [18, 1, 32]. Azimuthal modes can appear as standing or turning (also referred to as spinning) modes and both are observed in gas turbines. Bifurcations between standing and turning modes may be due to non-linear effects: $[3,32]$ propose a non-linear theoretical approach showing that standing wave modes can be found at low oscillation amplitudes but that only one turning mode is found for large amplitude limit cycles. Other explanations can be found in linear approaches: turning modes would appear only in perfectly axisymmetric configurations while any symmetry modification would lead to standing modes [33, 34]. [20] suggest that the initial flow conditions might trigger either standing or turning modes and $[32,2]$ observe that turbulence can cause random mode switching between standing and turning structures. Note that experimentalists also observe standing modes which have a structure that slowly rotates, e.g. the pressure nodes, will turn around the chamber at a low velocity (typically a convective velocity). We will call these modes 'rotating' and show that they are present in our LES (Table 1).

Validating any theory or acoustic method in the framework of annular chambers remains a challenge: only a few experiments have been conducted in labo- 
ratories (T.U. Munich, EM2C Paris) to study azimuthal modes in full annular chambers because multiburner combustion chamber rigs are expensive and offer limited optical access. A solution was proposed by [35] who studied an azimuthal system of Rijke tubes where flames are replaced by electric heaters. An alternative path is to rely on numerical simulation: massively parallel Large Eddy Simulation (LES), first developed for simple flames [36, 37, 38, 10, 39], have been used recently for full annular chambers [22, 40, 41] despite the very high computational cost. The main advantage of LES is that it relies on fewer modelling assumptions than acoustic models and can correctly predict flame / flame interactions, limit cycles, wall effects, chemical effects, etc.

An important aspect of all combustion instability studies is control: how can the combustor design be changed to avoid instability or at least mitigate its level? Fully active combustion instability control [42, 43, 44] has been successfully tested for azimuthal instabilities on certain industrial gas turbines [45] but its cost and its difficult certification for aero engines make it less attractive today than trying to understand and avoid azimuthal modes and building intrinsically stable combustors. Developing an LES which captures unstable azimuthal modes can then be used to understand the source of the instability and look for passive stabilisation methods at a reasonable cost.

The present paper describes a LES of azimuthal modes in a realistic helicopter annular combustion chamber. The reacting unsteady flow in a full annular chamber containing fifteen sectors is computed using massively parallel computers: this compressible simulation uses the exact chamber geometry, a surrogate fuel model for kerosene, a high-order numerical scheme and a fully compressible solver to track acoustic waves. It captures the self-excited azimuthal modes appearing in the real engine and provides unique insight into the behaviour of the unstable combustor: the LES reveals that the dominant mode is a standing azimuthal mode (Type 1 in Tab. 1) submitting sectors located at axial velocity antinodes to strong flow oscillations and periodic flashbacks. This mode can also transition to a turning mode (Type 2) for a few cycles and indicators of the mode nature (Tab. 1) show that during the 100 cycles com- 
puted with LES, approximately 20 cycles exhibit a turning (Type 2) structure while the remaining 80 cycles correspond to Type 3 (standing mode rotating at a convective velocity). The transition from Type 2 to 3 seems to be caused by turbulent fluctuations. LES results are also used as the baseline 'numerical experiment' to test other methods. For example, an acoustic solver [46, 30] is applied to the same configuration and results are compared. The acoustic solver requires FTFs of single sector flames as input: these FTFs are computed using LES of a single-sector of the same combustor that also provides fields of average sound speed. The acoustic solver can provide guidelines to control the instability: in the present case, it suggests that the azimuthal mode can be damped by decreasing the delay of the FTF of a single burner. This is tested successfully in the LES of the full combustor by increasing the preexponential constant of the chemical reaction. Even though the mean flame is moderately affected by the faster chemistry, the unstable azimuthal mode is totally damped and dies in 5 to 8 cycles, confirming that the acoustic solver captures the instability source and can be used to damp it.

The numerical tools (LES and acoustic solvers) are described in Section 2. The configuration is presented in Section 3. The LES results for an unstable mode are presented in Section 4 and the structure of the modes is studied in Section 5. The transfer function and the Rayleigh criterion are discussed in Section 6 and Section 7 before presenting the results of the acoustic solver in Section 8: a stability map is obtained in terms of FTF delay and shows that the unstable mode should be damped when the delay is reduced below a critical value $\tau_{0}$. This is done in Section 8.2 where a faster chemistry is used in the LES and the mode is damped. Appendix Appendix A discusses one important issue linked to the rest of the work: the specification of acoustic impedances at the inlet and outlet of the combustor. 


\section{Description of the numerical tools}

Two numerical tools are needed to investigate combustion instabilities in complex geometry chambers:

- A Large Eddy Simulation (LES) code to explicitly resolve all flame movements in the time domain (Section 2.1)

- An acoustic solver based on frozen mean flow, which provides the frequency and structure of all modes (Section 2.2)

The LES is much more costly than the acoustic computations and very few LES of full azimuthal chambers have been reported up to now [22, 40, 41]. Combining LES results with the outputs of an acoustic solver allows to further analyse and understand the LES data.

\subsection{LES solver}

The LES solver is a fully unstructured compressible code, including species transport and variable heat capacities [47, 38, 48]. Centered spatial schemes and explicit time-advancement are used to control numerical dissipation and capture acoustics. For the present case, a third order accurate in space and time scheme is employed with a time step limited by the speed of sound, namely the TTGC scheme [49]. The sub-grid scale viscosity $\nu_{t}$ is defined by the classical Smagorinsky model [50], $\nu_{t}=\left(C_{S} \Delta\right)^{2}\|S\|$, where $\Delta$ and $S$ are respectively the the filter characteristic length (approximated by the cubic root of the cell volume) and the resolved strain tensor. Sub-grid thermal and molecular fluxes are modelled using an eddy diffusion assumption with constant sub-grid Prandtl (0.9) and Schmidt (0.9) numbers, respectively.

Characteristic NSCBC boundary conditions [51, 47] are used for inlets and outlets. Walls are handled with a law-of-the-wall formulation [38]. Multiperforated plates are effusion-cooled walls and may be taken into account using a homogeneous model $[52,53]$. 
The LES solves the compressible Navier-Stokes equations given as

$$
\frac{\partial \overline{\mathbf{w}}}{\partial t}+\nabla \cdot \overline{\mathbf{F}}=\overline{\mathbf{s}}
$$

where $\overline{\mathbf{w}}$ is the vector of gaseous conservative variables, $\overline{\mathbf{F}}$ is the flux tensor composed of viscous, inviscid and subgrid scale components and $\overline{\mathbf{s}}$ is the vector of source terms. $\overline{\mathbf{w}}$ and $\overline{\mathbf{s}}$ are given respectively by:

$$
\begin{gathered}
\overline{\mathbf{w}}=\left(\bar{\rho} \tilde{u}, \bar{\rho} \tilde{v}, \bar{\rho} \tilde{w}, \bar{\rho} \tilde{e}, \bar{\rho} \tilde{Y}_{k}\right) \\
\overline{\mathbf{s}}=\left(0,0,0, \overline{\dot{\omega}}_{T}, \overline{\dot{\omega}}_{k}\right)
\end{gathered}
$$

In Eq. (2), $\bar{\rho}$ is the density, $(\tilde{u}, \tilde{v}, \tilde{w})$ are the velocity components, $\tilde{e}=\tilde{e}_{s}+$ $1 / 2 \tilde{u}_{i} \tilde{u}_{i}$ is the total non chemical energy ( $\tilde{e}_{s}$ is the sensible energy) and $\tilde{Y}_{k}$ are the species mass fractions. Five independent species are solved here $(k=5)$ : $J P 10, \mathrm{O}_{2}, \mathrm{~N}_{2}, \mathrm{CO}_{2}$ and $\mathrm{H}_{2} \mathrm{O} . \tilde{f}$ denotes Favre filtering. In Eq. (3), combustion terms are the reaction rate $\overline{\dot{\omega}}_{k}$ and the heat release $\overline{\dot{\omega}}_{T}$, their modeling is described hereafter.

In the following LES calculations, kerosene is replaced by a synthetic surrogate: JP10, which has very similar properties. The reaction rate $\overline{\dot{\omega}}_{k}$ is modelled by a one-step irreversible Arrhenius law [10] with coefficients fitted from detailed chemistry (composed of 43 species and 174 steps) [54]: $J P 10+14 \mathrm{O}_{2} \rightarrow$ $10 \mathrm{CO}_{2}+8 \mathrm{H}_{2} \mathrm{O}$, using criteria based on laminar flame speed and temperature [55]. The progress rate of the reaction is written as

$$
\dot{\mathcal{Q}}=A\left(\frac{\rho Y_{C_{10} H_{16}}}{W_{C_{10} H_{16}}}\right)^{1.5}\left(\frac{\rho Y_{O_{2}}}{W_{O_{2}}}\right)^{0.55} \exp \left(-\frac{T_{a}}{T}\right)
$$

Reduced one-step schemes guarantee proper flame speed predictions only in the lean regime (i.e. with equivalence ratios $\Phi<1$ ). Such a chemical scheme is not sufficient for the target configuration since the local equivalence ratio reaches a wide range of values. To circumvent this shortcoming, the pre-exponential constant of the one-step scheme $A$ is adjusted as a function of the local equivalence ratio $\Phi$ to reproduce the proper flame speed dependency on the rich side $[56,55,57]$. 
To handle flame/turbulence interactions in this partially premixed flame, the dynamically thickened flame model (TFLES) is used [58, 49, 38]. This model detects reaction zones using a sensor and thickens the flame front by a factor $F$ in these zones so that it can be solved on the LES grid. The interaction between turbulence and chemistry at the subgrid level is modelled by an efficiency function $E$ which compensates the effect of thickening and accounts for the influence of turbulence on the subgrid turbulent flame speed $[58,59]$.

\subsection{Helmholtz solver}

A 3D parallel Helmholtz solver in the frequency domain is used to predict eigenfrequencies and mode structure assuming that the mean flow is frozen and that the flame's effect on the acoustic field can be represented through a transfer function between velocity and heat release fluctuations [60]. It solves the eigenvalue problem of a Helmholtz equation with a source term due to the flames arising from a discretisation on unstructured meshes [30].

Starting from the linearised conservation equations for mass and momentum in a reactive non-viscous low-Mach flow, one obtains [10]

$$
\begin{gathered}
\frac{1}{c_{0}^{2}} \frac{\partial p_{1}(\mathbf{x})}{\partial t}+\rho_{0} \nabla \mathbf{u}_{1}(\mathbf{x})=\frac{\gamma-1}{c_{0}^{2}} \dot{\omega}_{1 T} \\
\frac{\partial \mathbf{u}_{1}(\mathbf{x})}{\partial t}+\frac{1}{\rho_{0}} \nabla p_{1}(\mathbf{x})=0
\end{gathered}
$$

where $\rho_{0}$ is the mean density and $c_{0}$ the sound speed, $p_{1}$ is the acoustic pressure fluctuation, $\mathbf{u}_{1}$ the acoustic velocity and $\dot{\omega}_{1 T}$ the heat release fluctuation.

Assuming harmonic fluctuations, the time-variation of a quantity $f$ can be expressed as $f_{1}=f^{\prime} e^{-j \omega t}$. Combining Eq. 5 and Eq. 6 one yields the Helmholtz equation, solved in the frequency domain for the pressure oscillation amplitude $p^{\prime}$ at $\omega=2 \pi f$ :

$$
\nabla \cdot c_{0}^{2}(\mathbf{x}) \nabla p^{\prime}(\mathbf{x})+\omega^{2} p^{\prime}(\mathbf{x})=j \omega(\gamma-1) \dot{\omega}_{T}^{\prime}
$$

At this point, the equations cannot be closed without an additional assumption allowing to express the unsteady reaction rate $\dot{\omega}_{T}^{\prime}$ as a function of the 
unsteady pressure. The most classical approach is to introduce a Flame Transfer Function (FTF) linking $\dot{\omega}_{T}^{\prime}$ to velocity perturbations (which can be obtained from the pressure gradient) at a given reference point in the burner. The FTF relates the velocity fluctuations upstream of the flame to the fluctuations of the heat release via an interaction index $n_{u}(\mathbf{x})$ and a time delay $\tau_{u}(\mathbf{x})$ [30]:

$$
\frac{\dot{\omega}^{\prime} T}{\dot{\omega}_{T}}=n_{u}(\mathbf{x}) \frac{\mathbf{u}^{\prime}\left(\mathbf{x}_{\mathrm{ref}}, t-\tau_{u}(\mathbf{x})\right) \cdot \mathbf{n}_{\text {ref }}}{U_{b u l k}}
$$

where $\mathbf{x}_{\mathbf{r e f}}$ is a reference point, located just upstream of the flame, where velocity fluctuations are measured.

This formulation must be extended for annular combustion chambers that may be composed of ten to twenty burners. The unsteady reaction rate in each sector $i$ of the annular combustor is supposed to depend only on the velocity fluctuations at the reference point $\mathbf{x}_{\text {ref }}$ of this sector. There is one single reference point for each sector and therefore 15 for the whole combustor. Each point $\mathbf{x}$ in the combustor belongs to a sector $i$ and has an associated reference point $\mathbf{x}_{\mathbf{r e f}}(\mathbf{x})$ in this sector. An important assumption used implicitly in the FTF approach for annular chambers (and in all models found in the literature) is the ISAAC (Independence Sector Assumption in Annular Combustors), introduced by $[22,61]$. ISAAC states that the heat release fluctuations in a given sector are driven only by the fluctuating mass flow rates in the swirler of this sector, which implies that flames do not interact in the chamber and that the only mechanism leading to unsteady combustion is the fluctuating longitudinal velocity through the swirlers. ISAAC is probably not satisfied in all combustors, such as when flame injectors are close to each other [62] or when flame / flame interactions are important. However, in most gas turbine chambers, ISAAC seems to be satisfied and it will actually be verified here using LES (Section 6).

The final Helmholtz equation solved for the pressure fluctuation is:

$$
\nabla \cdot c_{0}^{2}(\mathbf{x}) \nabla p^{\prime}(\mathbf{x})+\omega^{2} p^{\prime}(\mathbf{x})=\frac{(\gamma-1)}{\rho_{0}(\mathbf{x})} n_{u, i}(\mathbf{x}) e^{j \omega \tau_{u, i}} \nabla p^{\prime}\left(\mathbf{x}_{\mathbf{r e f}}(\mathbf{x})\right) \cdot \mathbf{n}_{\text {ref }}
$$

Equation 9 is the general form used in this study but it can be simplified when flames are compact. For these flames, the acoustic wavelength is much 
longer than the flame length and the time delay of Eq. 8 can be assumed to be constant: $\tau_{u}(\mathbf{x})=\tau$. In such a situation, the Helmholtz solver can be used to construct stability maps as a function of the single global delay $\tau$ and this property will be used in Section 8.1.

\section{Configuration}

The combustion chamber in studied case contains fifteen burners (Fig. 1). To avoid uncertainties associated with the boundary conditions, especially on inlet and outlet impedances, the chamber casing is also computed. The computational domain starts after the inlet diffuser and ends at the throat of the high-pressure stator, which is replaced by a choked nozzle with the same minimum section, so that the flow in the outlet zone and the acoustic reflection at the throat are explicitly computed by the solver. The air and fuel inlets use non-reflective boundary conditions [51]. The air flowing in the casing feeds the combustion chamber through the swirlers, cooling films and dilution holes, which are all explicitly meshed and resolved (Fig. 1). Walls are treated with a law-of-the-wall approach and multi-perforated plates are modelled by a homogeneous model $[52,53]$. Pure fuel (JP10) is injected at the outlets of the swirlers through a circular slit of $2 \mathrm{~mm}$ width, with a velocity of $5 \mathrm{~m} / \mathrm{s}$ (Fig. 1). The flow is supposed to be gaseous to avoid complexities associated with atomisation and evaporation. Six different unstructured meshes are used in this study: a single sector (i.e. only one burner) LES grid, three full annular combustor LES grids (15 sectors) and a coarse annular grid designed for Helmholtz calculations (Tab. 2).

The reacting flow in the combustor of Figure 1 is simulated by first computing a single sector, duplicating the result 14 times around the turbine axis (Fig. 2), and then letting the computation evolve to the most amplified oscillation mode. No forcing is added: the LES captures (if present) the oscillation modes of the combustor without any external excitation [22]. 


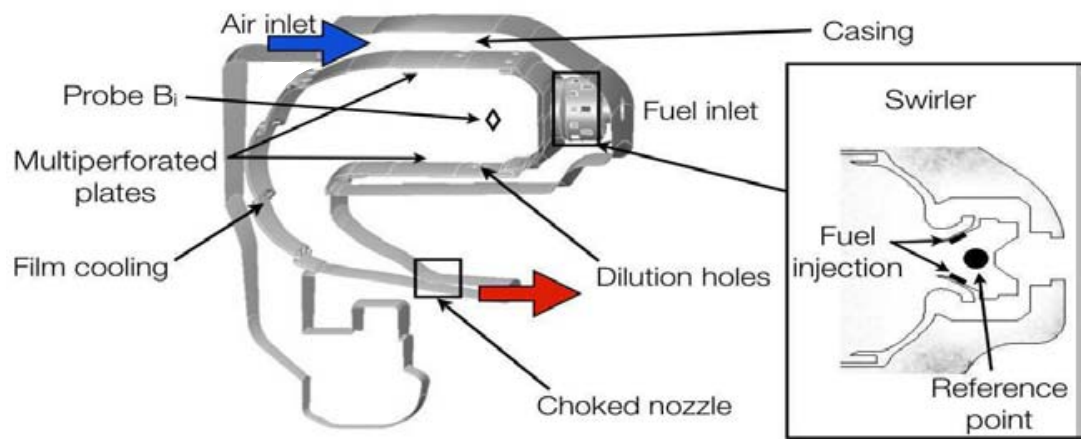

Figure 1: Gas turbine geometry shown on a single sector. Two points of special interest are displayed: Probe $B_{i}$ and the reference point $\mathbf{x}_{\mathbf{r e f}, \mathbf{i}}$ of the $n-\tau$ model used by the Helmholtz solver.

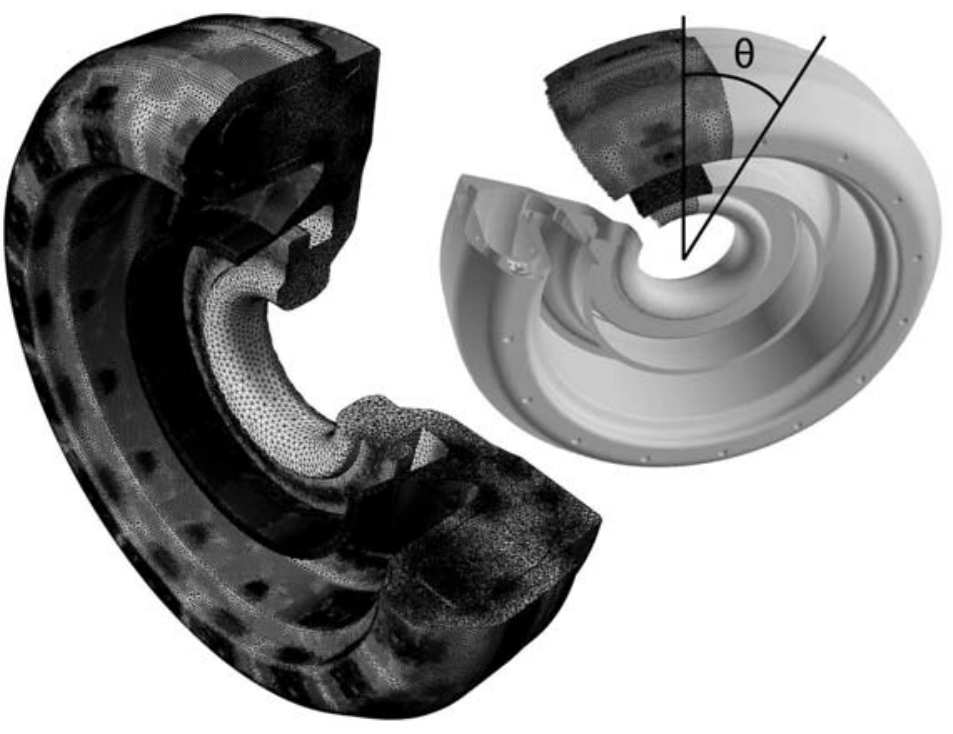

Figure 2: Geometry along with the mesh. 


\begin{tabular}{ccc}
\hline Domain & Number of points & Number of tetrahedral cells \\
\hline Single sector LES & 518649 & 2819176 \\
\hline Full annular LES & & \\
Coarse & 7694265 & 42287640 \\
Medium & 16466145 & 93147720 \\
Fine & 54954975 & 336078255 \\
\hline Full annular Helmholtz & 362640 & 1684860 \\
\hline
\end{tabular}

Table 2: Domains and grids: the first five domains are used for LES, Helmholtz simulations are carried out with the last one.

\section{Large Eddy Simulation of azimuthal modes}

In most cases, LES results reveal a transient period of growth followed by the formation of azimuthal modes in the combustion chamber: Fig. 3 displays the azimuthal velocity measured by a probe located in a zone of the casing where no significant azimuthal flow should exist. This velocity component starts oscillating promptly. A time-frequency analysis, based on wavelet transform, is performed to analyse the apparition of the mode [63]. Figure 4 shows the time evolution of the frequency content of the pressure fluctuation signal measured at probe $B_{1}$. At the very beginning of the simulation, a frequency of $500 \mathrm{~Hz}$ is found and quickly vanishes, soon replaced by a frequency of $750 \mathrm{~Hz}$. This stems from the fact that a longitudinal mode at $500 \mathrm{~Hz}$ is present in the single sector calculation. This longitudinal mode disappears and azimuthal modes around $750 \mathrm{~Hz}$ settle when including all the burners in the simulations. These modes cause the flames to oscillate both azimuthally and longitudinally, creating periodic flashbacks inside the injectors, as illustrated by Figure 5 (or in the animation available at elearning.cerfacs.fr/combustion/illustrations/azimut/index.php). Figure 6 displays the pressure fluctuation spectrum of the probe $B_{1}$ located in the combustion chamber and reveals a distinct peak at $750 \mathrm{~Hz}$. The mesh independence of the LES was checked on a massively parallel system [31, 64] by comparing LES obtained on the three meshes with 42, 93 and 330 millions of 


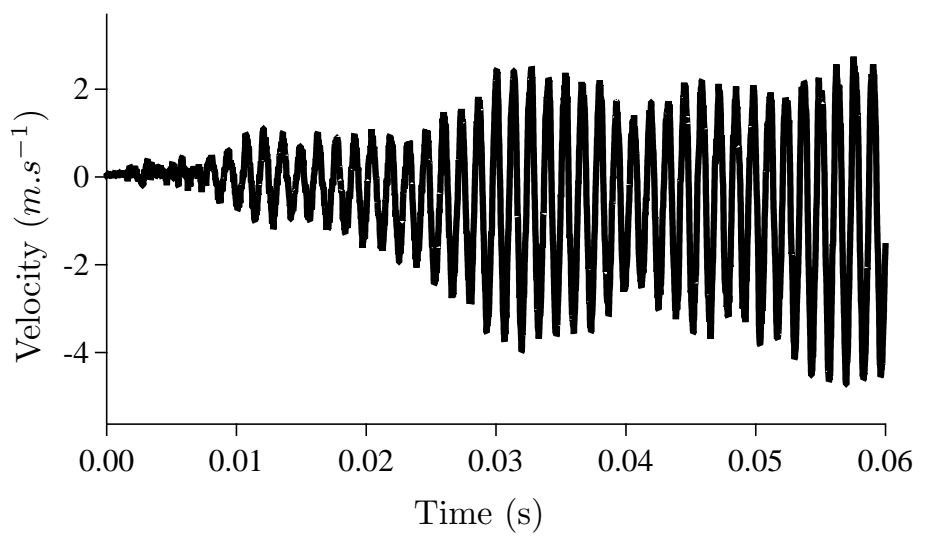

Figure 3: Azimuthal velocity measured by a probe located in a quiet zone of the casing.

cells. Results were very similar: the mode structure was the same so that only the 42 million cells mesh is considered here.

\section{Mean swirl, spinning and standing modes}

\subsection{A simple model for azimuthal waves}

Even though the configuration of the swirlers is axi-symmetric, the swirl direction is the same in each burner and makes one rotation direction preferential, leading to the existence of a mean swirling velocity $V_{\theta}$ in the whole annular combustor. The azimuthal mode that forms in the chamber is primarily composed of two waves traveling in different directions. These two components induce a velocity difference between the co-spinning wave (spinning in the direction of the swirl induced by the injectors), called here the " +" wave, and the counterspinning one, called the "-" wave. To first order, the + wave turns at a velocity $c+V_{\theta}$ where $V_{\theta}$ is the mean swirl velocity and $c$ the mean sound speed in the chamber while the - wave turns at $c-V_{\theta}$. The mean swirling velocity $V_{\theta}$ is small compared to the sound speed $c$, average typical swirl velocities of $10 \mathrm{~m} / \mathrm{s}$ are observed in the LES. This allows the effect of the sound speed to be separated from the effect of the swirl velocity, the main azimuthal mode is observed at a 


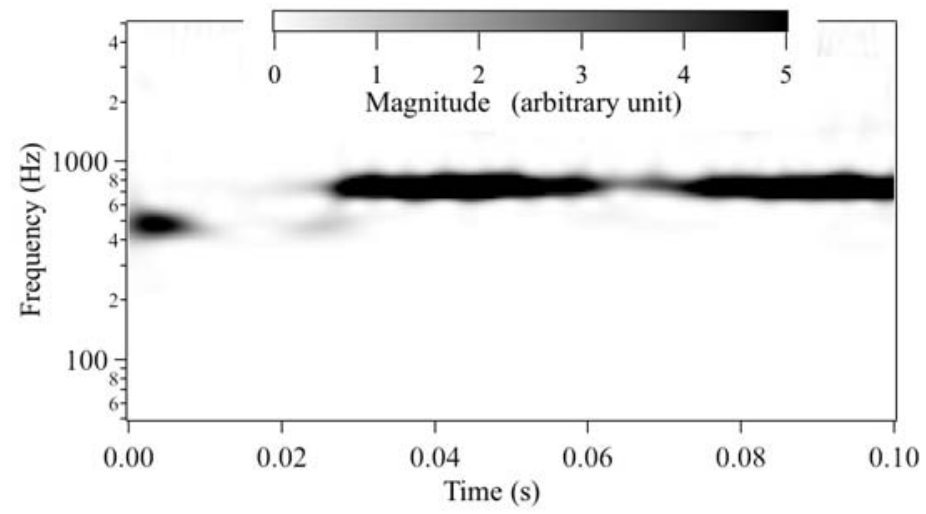

Figure 4: Time-frequency analysis for the pressure fluctuations measured at probe $B_{1}$ (Fig. 1).

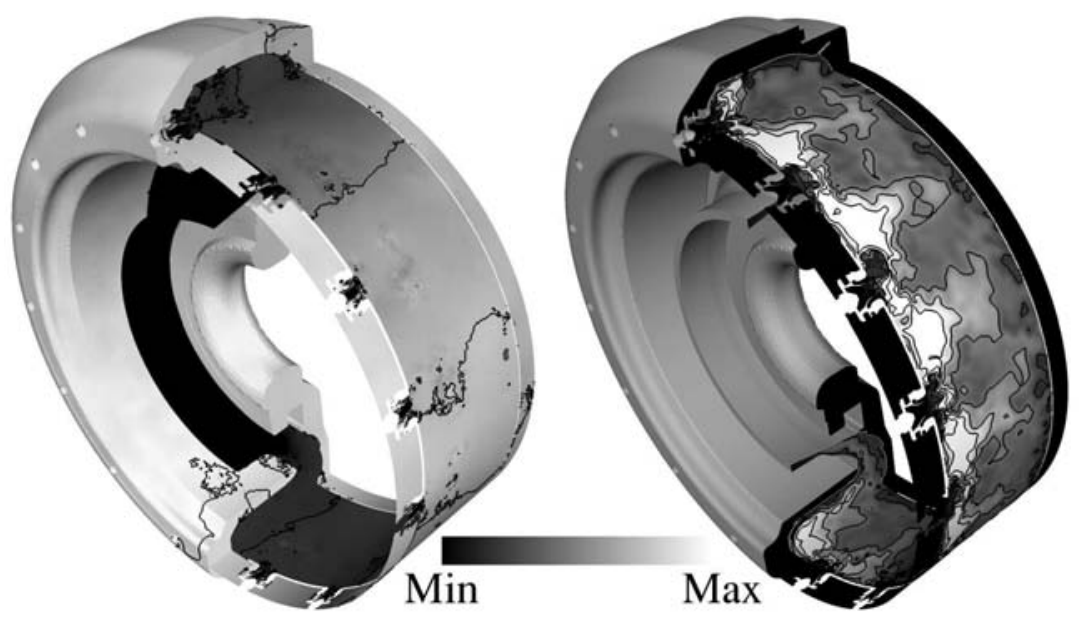

Figure 5: Flow visualisation. Left: instantaneous pressure field with pressure contours. Right: temperature field with temperature contours on a cylindrical plane passing through the $B_{i}$ probes. 


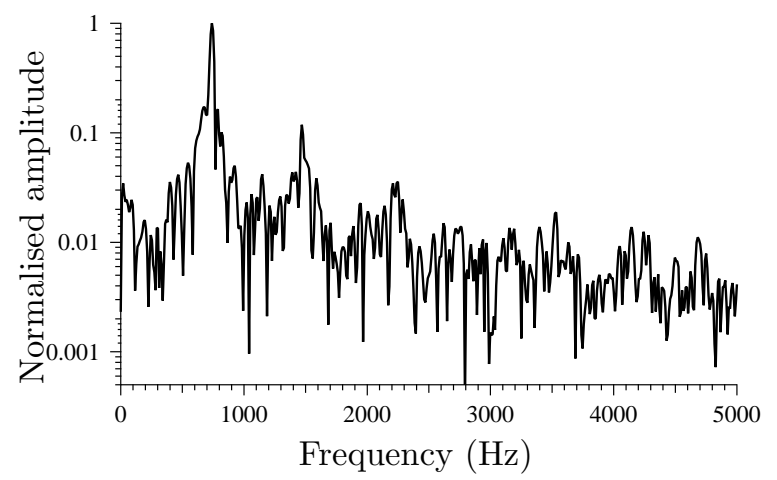

Figure 6: Pressure fluctuation spectrum of the $B_{1}$ probe located in the combustion chamber.

carrier frequency of the order of $c / 2 \pi R$ and is modulated by a lower frequency of the order of $V_{\theta} / 2 \pi R$.

To illustrate this point and ease the interpretation of LES results in the following, a simple model is described here to express the pressure oscillations $p_{1}$ resulting from the combination of the + and - waves in an annulus of radius $R$ where the period and the angular frequency of the azimuthal mode without swirling flow are $T_{a z i}=2 \pi R / c=2 \pi / \omega$ and $\omega=c / R$, respectively. The pressure signal may then be written as

$$
p_{1}=p^{\prime} e^{-j \omega t}=\left[A_{+} e^{j\left(\theta-V_{\theta} t / R\right)}+A_{-} e^{j\left(-\theta+V_{\theta} t / R\right)}\right] e^{-j \omega t},
$$

where $\theta$ is the angle measuring a point position along the azimuthal direction (Fig. 2). Note that for a perfectly standing mode (Type 1 in Table 1) $A_{+}=A_{-}$ and $V_{\theta}=0$, for a turning mode (or spinning mode, Type 2) $A_{-}$or $A_{+}=0$ and $V_{\theta}=0$, and for a rotating mode (Type 3) $A_{+}=A_{-}$and $V_{\theta} \neq 0$. A dependence on the axial direction $x$ is possible $[18,20]$ but is not included here, this simplification will be verified in Section 5.2. The $V_{\theta} t / R$ terms are induced by the mean swirl convection at speed $V_{\theta}$. They change very slowly compared to the $\omega t$ term so that a structure can be defined for $p_{1}$ by observing it over a few periods of the short (acoustic) time: this structure then changes over long (convective) times. Typically, in gas turbines one can observe standing 
azimuthal modes (oscillating at hundreds of Hertz) where the pressure nodes are rotating very slowly (depending on the configuration, one full rotation could take a few seconds to as long as a few hours). The period required for a complete rotation of the structure is $2 \pi R / V_{\theta}$ or $T_{a z i} / M_{a}$, where $M_{a}=V_{\theta} / c$ is the Mach number of the swirling flow convective component.

This makes the analysis of azimuthal modes more complicated: a standing mode (observed over a few periods) can exhibit a structure which rotates slowly (with the swirl velocity). Such a mode (Type 3 in Table 1) is not a spinning mode (Type 2) where the pressure field turns with the sound speed and $p_{1}$ would be written as $p_{1}=A e^{j(\theta-\omega t)}$. When using LES, sampling over very long times is difficult, and hence observing a full rotation of such a structure is costly. In the present LES, the oscillations were computed for $153 \mathrm{~ms}$ corresponding to 115 cycles of the azimuthal mode but only slightly more than one rotation of the rotating structure at $V_{\theta}$. However, as soon as the rotation effect due to the mean swirl component has been identified, the structure can be studied over a few periods of the azimuthal mode, knowing that it will rotate with the mean swirl velocity $V_{\theta}$ if one observes it for a long time. This rotation does not affect the fundamental mode structure observed at shorter times.

As an example, Figure 7 shows the mode structure (modulus and phase of $\left.p_{i}^{\prime 1}\right)$ computed using Eq. 10 at two instants $t_{i}(i=1,2)$ separated by 25 periods of the azimuthal mode $T_{a z i}$ in a case where $A_{+}=1, A_{-}=0.95$ and $V_{\theta}=10$ $\mathrm{m} / \mathrm{s}$ :

$$
p_{i}^{\prime}=A_{+} e^{j\left(\theta-V_{\theta} t_{i} / R\right)}+A_{-} e^{j\left(-\theta+V_{\theta} t_{i} / R\right)},
$$

where $t_{2}=t_{1}+25 T_{a z i}$. As expected, the mode keeps the same structure, very similar to a standing mode, but it has rotated slowly between the two instants, shifting the angular position of pressure nodes and antinodes by approximately $\pi / 2$.

\footnotetext{
${ }^{1}$ The exact expression for the phase $\phi_{1}$ plotted in Figure 7 is the difference between the argument of $p_{1}$ (which depends on $t$ in Eq. 10 ) and its value at a fixed $\theta_{0}$ which is fixed here to $\theta_{0}=0$. The plotted phase is $\phi_{1}=\arg \left(p_{1}\right)-\arg \left(p_{1}(\theta=0)=\arg \left(p_{1}\right)+\omega t=\arg \left(p^{\prime}\right)\right.$.
} 

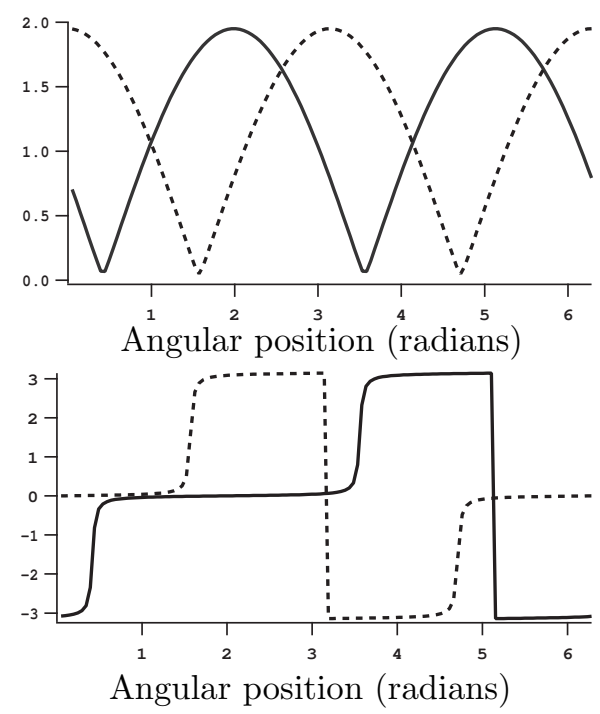

Figure 7: Pressure perturbations modulus (left) and phase (right) versus the angle at two times separated by a time equal to 25 periods of the azimuthal mode in an ideal annular chamber with $R=0.176 \mathrm{~m}$ (radius), $c=790 \mathrm{~m} / \mathrm{s}$ (sound speed) and $V_{\theta}=10 \mathrm{~m} / \mathrm{s}$ (average swirl velocity) (Eq. 10). 


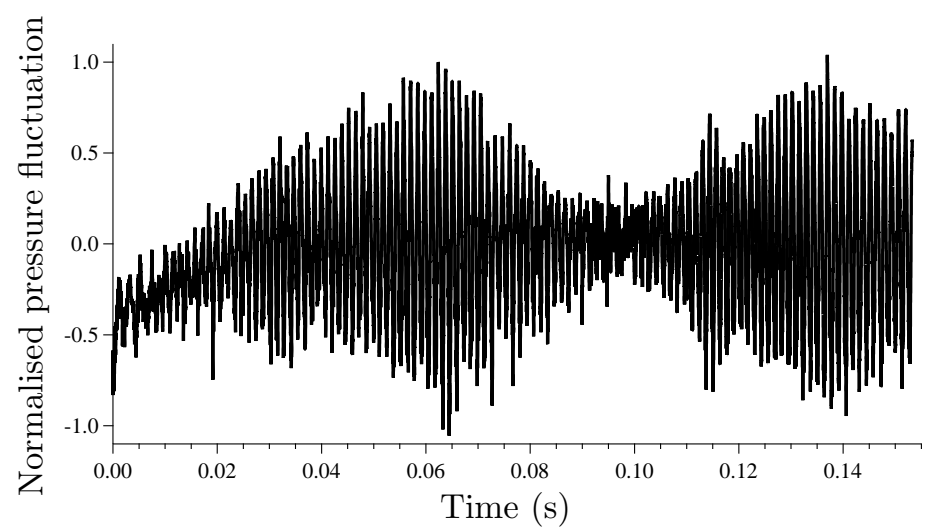

Figure 8: Pressure fluctuations versus time at probe $B_{12}$.

\subsection{Analysis of modes observed in LES}

LES results are analysed here over a large number of cycles (100) to investigate the mode structure. The time evolution of the pressure fluctuations at probe $B_{12}$ is displayed in Fig. 8. As expected from Eq. 10, the pressure oscillates at a frequency of $750 \mathrm{~Hz}$ modulated at a much lower frequency. Figure 9 displays the mode structures obtained at two instants of the simulation $t_{1}=0.143 \mathrm{~s}$ and $t_{2}=0.153 \mathrm{~s}$. To construct these structures, seven cycles at $750 \mathrm{~Hz}$ are sufficient. Figure 9 demonstrates that, at this moment of the simulation, a standing mode is observed and that this standing mode is rotating slowly (Type 3). The rotation velocity is $44 \mathrm{rad} / \mathrm{s}$ corresponding to a mean swirl velocity $V_{\theta} \approx 7.8 \mathrm{~m} / \mathrm{s}$ in the combustion chamber. This value matches the levels measured in the LES.

Figure 9 can be used to compare full LES data (symbols in Fig. 9) to the output of the simple analytical model of Eq. 10 (lines in Fig. 9). In the present case, Eq. 10 matches LES results if $A_{-} / A_{+}=0.96$. Despite its simplicity, Eq. 10 captures almost perfectly the structure of the mode found in the LES (Fig. 9). This standing mode is rotating slowly because of the mean swirl produced in the chamber, as predicted by Eq. 10. No purely spinning mode (for which either $A_{+}$or $A_{-}$must be zero, Type 2) is observed at these instants. [32] suggest that standing modes are less likely to be found than spinning modes when the limit cycle is reached, whereas [20] imply that branching between 

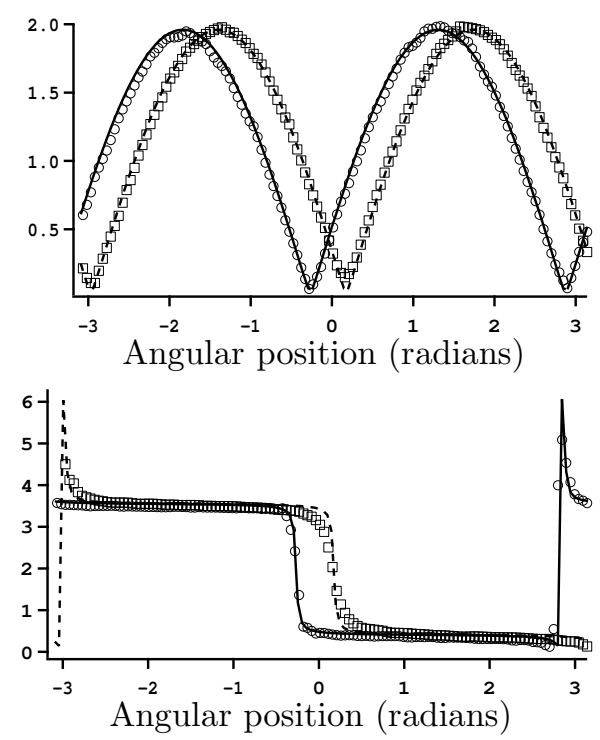

Figure 9: LES results: pressure perturbations modulus (left) and phase (right) at two times $t_{1}=0.143 \mathrm{~s}$ (circles) and $t_{2}=0.153 \mathrm{~s}$ (squares). A fit using Eq. 10 is also added with $A_{-} / A_{+}=0.96$ (solid and dashed lines).

standing or spinning modes might depend on the initial flow field. [34, 2] predict standing modes in strongly asymmetrical configurations, purely spinning modes in symmetrical ones and mixed modes in between. They also mention that an initially standing mode is likely to become a spinning mode when the limit cycle is reached and that the transient duration significantly varies, depending on the initial conditions. The model used in these studies considers small perturbations and, while correctly predicting the stability nature of the system, leads to mixed standing/spinning modes when including large perturbations. All these models are based on assumptions about the mode structure and about the link between heat release and acoustics, which are absent in the present LES that explicitly resolves the unsteady flames. Both turbulent noise and azimuthal modes are captured by the LES: Figure 6 reveals high levels of background hydrodynamic noise that may invalidate the hypothesis of small perturbations used in most models. 


\begin{tabular}{lccc}
\hline Mode type & $\mathrm{C}(\mathrm{t})$ & $|C(t)|$ & Phase of $\mathrm{C}(\mathrm{t})$ \\
\hline Standing & $A \cos (\omega t)$ & $A|\cos (\omega t)|$ & constant \\
Right turning & $\frac{A}{2} e^{j \omega t}$ & $\frac{A}{2}$ & $\omega t$ \\
Left turning & $\frac{A}{2} e^{-j \omega t}$ & $\frac{A}{2}$ & $-\omega t$ \\
\hline
\end{tabular}

Table 3: Classification of modes and corresponding $\mathrm{C}(\mathrm{t})$ indicator.

Even though Fig. 9 shows a Type 3 mode (standing with a slow convectively rotating pattern) at times $t=0.143$ to $0.153 \mathrm{~s}$, other studies $[20,32,2]$ suggest that this could change with time. To investigate the mode nature over the whole LES duration, it is possible to use an indicator $C(t)$ as proposed by [65]. Let us consider an azimuthal mode written as in Eq. 10 and $N$ evenly distributed probes along $\theta$ (Fig. 10). A proper indicator of the mode type can be built as

$$
C(t)=\frac{1}{N} \sum_{k=1}^{N} p_{1}\left(\theta_{k}, t\right) e^{j \theta_{k}}
$$

where the angles $\theta_{k}$ are defined as shown in Fig. 10, the signal being measured by the corresponding $B_{i}$ probes.

The $C(t)$ indicator reveals the spinning nature of the mode [65]: a constant indicator modulus identifies a spinning mode, whereas an oscillating indicator modulus (at a $2 \omega$ pulsation) unveils a standing mode. Moreover, the phase of the indicator is constant for a standing mode and linearly increasing (or decreasing) with time for a right (respectively left) turning mode (see Table 5.2 and Appendix Appendix B for more details).

The time traces for modulus (Fig. 11) and phase (Fig. 12) of $C(t)$ confirm the presence of a standing mode, as discussed previously in Section 5, over most of the LES duration. However, a transition towards a mixed standing/spinning structure (Type 2) is found between $0.12 \mathrm{~s}$ and $0.135 \mathrm{~s}$ : the modulus of $C(t)$ shows reduced oscillation levels (Fig. 11) while its phase clearly unwraps like $-\omega t$ for a period of $15 \mathrm{~ms}$ (Fig. 12). Figure 13 compares the mode structures at $t=0.125 \mathrm{~s}$ and $t=0.143 \mathrm{~s}$ and validates the fact that the mode type evolves with time: at $0.125 \mathrm{~s}$, the mode is turning (Type 2), while at $0.143 \mathrm{~s}$ 


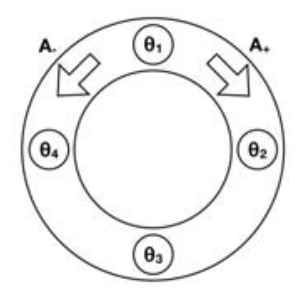

Figure 10: Angular location of the $B_{i}$ probes considered when building the $C(t)$ indicator, here shown for 4 angular locations.

it is an almost perfectly standing mode (Type 1). It has been experimentally reported that both spinning and standing modes are observed $[66,1,32]$ and the present LES exhibits the same behaviour. However, the underlying mechanisms driving this transition between mode types are still not clear. [20] observe the standing/spinning behaviour of modes in an axisymmetric annular combustor with 12 burners. Their low-order model approach [67] suggests that the initial flow conditions might trigger either standing or spinning modes. Frequency and stability of the mode are however found to be independent of its spinning nature. [32] and [2] observe that turbulence can cause random mode switching between standing and turning structures. The present work supports this observation but does not bring more insight into the reasons of this mode type change.

Some authors, such as $[18,67,20]$, include an axial component to the pressure waves described by Eq. 10. In the present case, Fig. 14 displays the longitudinal structure of the modes found by LES and predicted by the Helmholtz solver: it shows that the longitudinal dependency of the azimuthal mode is weak and can thus be neglected in this study. The peak that appears at the entrance of the chamber (normalised distance of 0.12 from the inlet) is not acoustic, but rather stems from the intense hydrodynamic activity in the swirlers and is not seen in the pressure amplitude predicted by the Helmholtz solver. Pressure fluctuations (Fig. 14) are normalised for both LES and acoustic solver so as to be of the same level in the chamber. The gap between both curves in the casing comes from differences in the normalisation due to the hydrodynamic content of the flow 


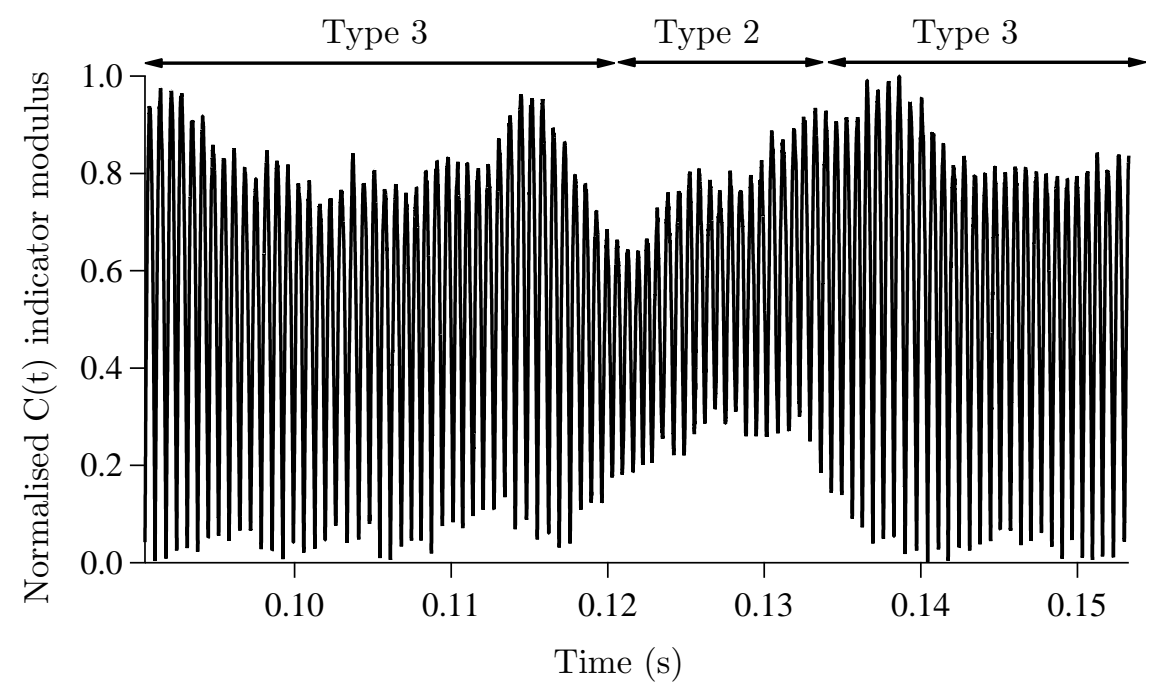

Figure 11: $C(t)$ indicator modulus, considering 12 probes evenly distributed.

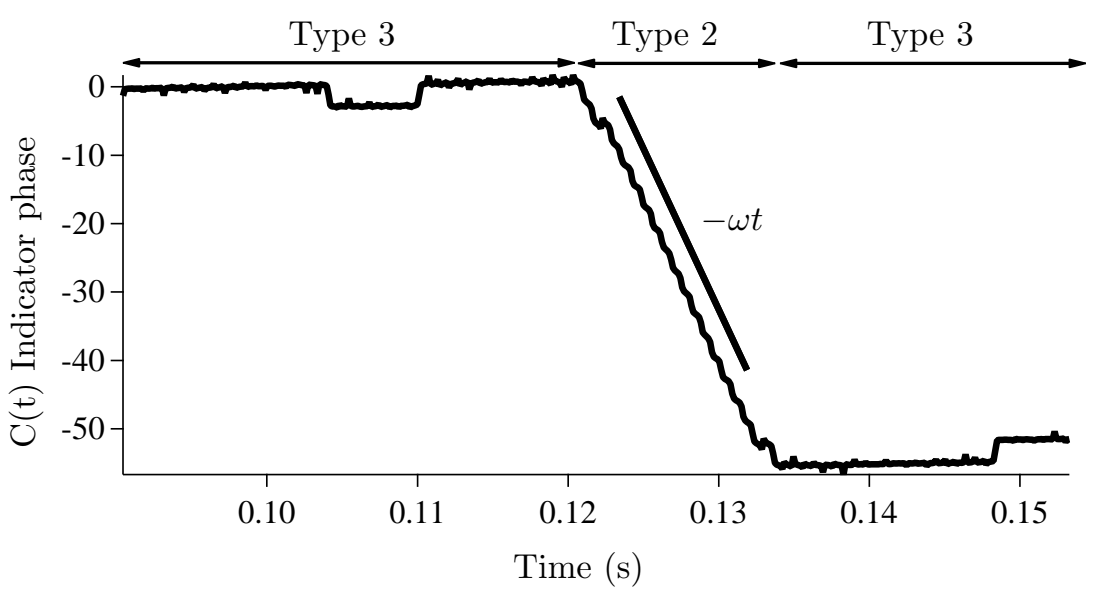

Figure 12: Phase of $C(t)$ indicator, considering 12 probes evenly distributed. 


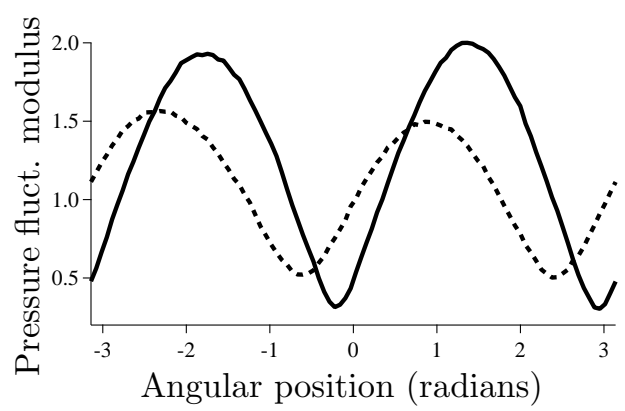

Figure 13: LES results: pressure perturbations modulus at two times $t=0.125 \mathrm{~s}$ (dotted line, mixed mode) and $t=0.143 \mathrm{~s}$ (continuous line, standing mode).
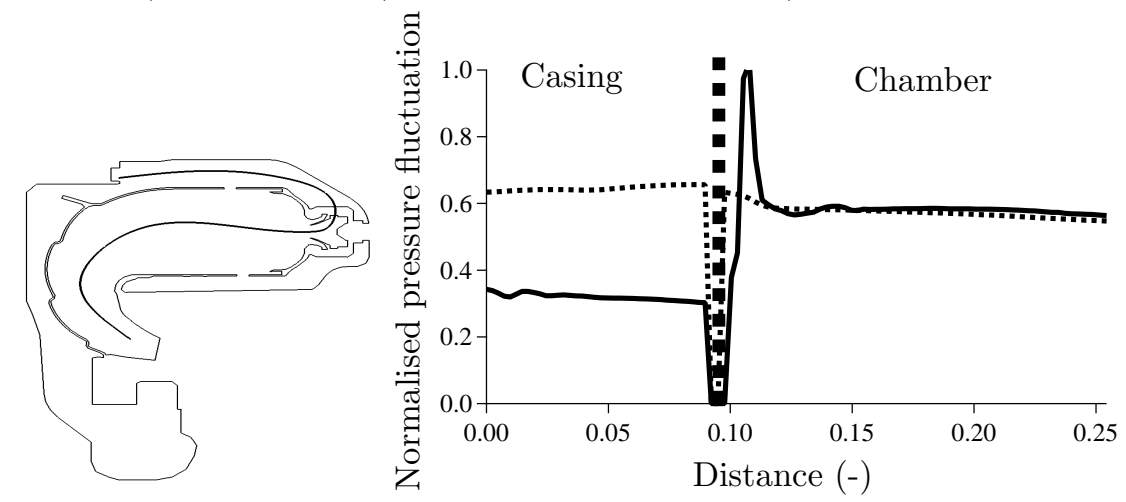

Figure 14: Longitudinal structure of the azimuthal mode in the LES (continuous line) and of the first azimuthal mode predicted by the Helmholtz solver (dotted line, see Section 8.1 and Fig ??). The structure is obtained with the measured or predicted values of the pressure fluctuations in the most fluctuating sector along the line shown on top of a sector geometry (left).

simulated by LES that is not present in the predictions of the Helmholtz solver.

\section{Transfer function}

Figure 15 displays instantaneous temperature fields at four different phase angles for two burners. These fields reveal a similar topology: the flames are stabilised by a recirculating zone created by the swirling motion imposed by the swirlers. However, during the oscillations observed in Fig. 8, the flames flashback periodically in the swirlers: the temperature field for the two burners 
displayed in Fig. 15 shows that the flames move during a period of the mode. The azimuthal mode is transverse (i.e. from left to right in Fig. 15) to the main direction of the flow and the flames indeed dance from left to right. This azimuthal movement is spectacular but has a limited effect on the instability. The important mechanism here is the perturbation of the axial flow rate through the swirlers, which is induced by the azimuthal mode in the chamber along $\theta$. This flow rate oscillation controls the reaction rate in front of the burner and is the source of the instability as expected in the ISAAC assumption introduced in Section 2.2: Fig. 16 displays variations of flow rate and heat release in various sectors, confirming that the unsteady reaction rate in each sector is controlled by the flow rate oscillations through the corresponding swirler. Moreover, the delay between flow rate and heat release oscillations is the same for all burners (Fig. 17) and is equal to the value obtained in a pulsated single burner LES, confirming again the validity of the ISAAC assumption and justifying its use in Section 8. These flow rate oscillations are the source of the strong back and forth axial displacement of the flames observed in Fig. 15: at some instants, they are repelled downstream from the swirler exit (e.g. at phase angle 2) and later enter the swirler (e.g. left flame at phase angle 3).

\section{Rayleigh criterion}

The Rayleigh criterion is a common condition used to assess thermo-acoustic stability [5]. It states that a necessary condition for thermoacoustic coupling is fulfilled if pressure and heat release fluctuations are in phase:

$$
\iiint_{V} p_{1} q_{1} d \mathcal{V}>0
$$

Most analytical models use assumptions to relate $p_{1}$ and $q_{1}$. LES is more general and can be used to assess the validity of these hypotheses. For example, in [2], a linear dependence between heat release and pressure fluctuations is assumed: $q_{1}=\beta p_{1}$. To check this assumption, phase-averaging of $p_{1}$ and $q_{1}$ was performed on the LES over 7 periods of the first azimuthal mode. 20 phase instants were 


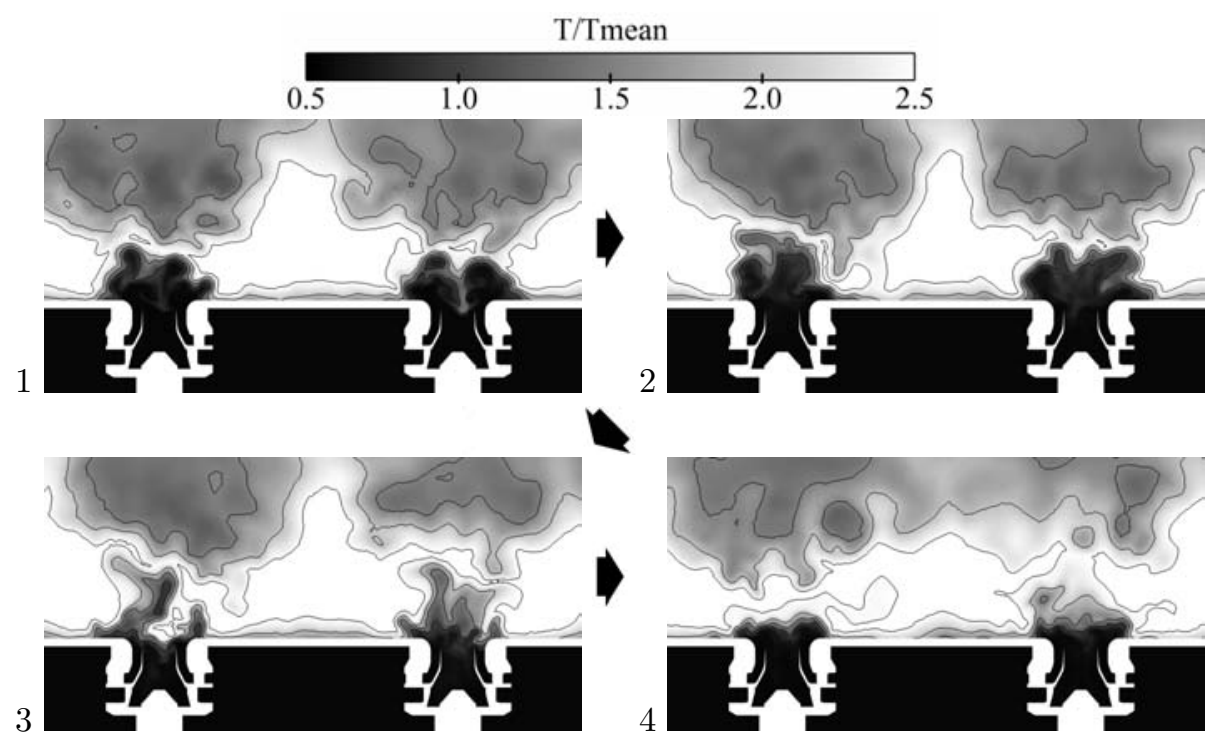

Figure 15: Instantaneous temperature field shown for 2 burners on a developed surface of a cylinder passing through the $B_{i}$ probes. Four phase instants are displayed: top left (0), top right $\left(\frac{\pi}{2}\right)$, bottom left $(\pi)$ and bottom right $\left(\frac{3 \pi}{2}\right)$.
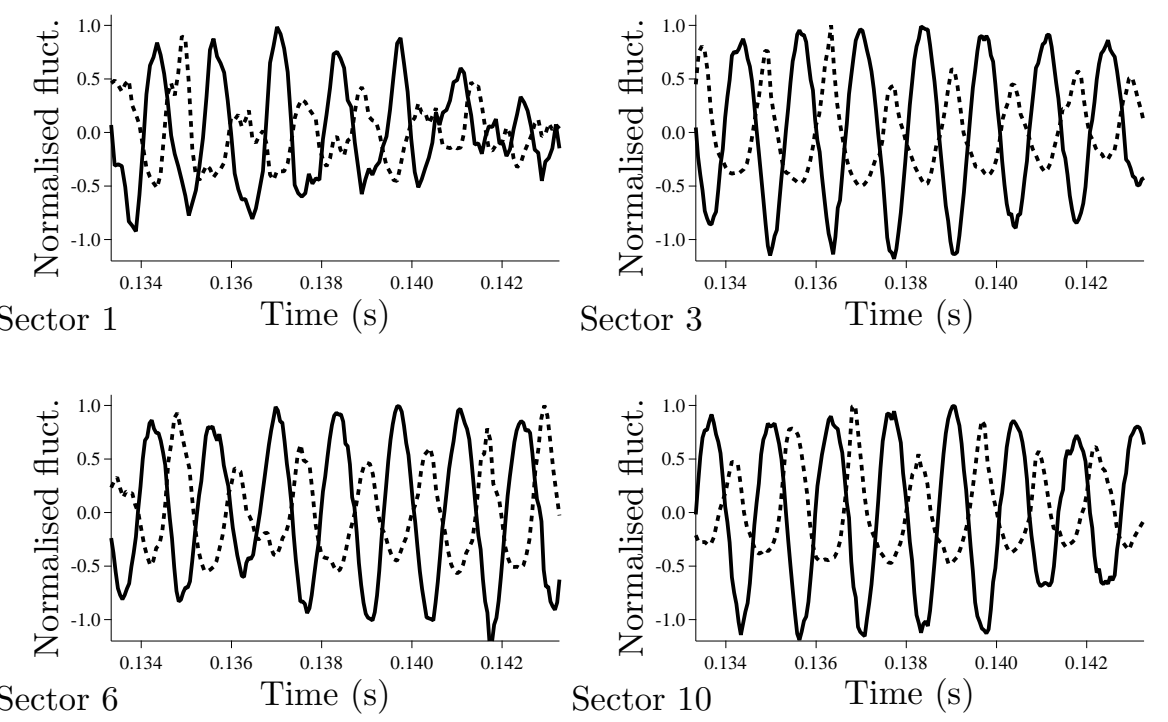

Figure 16: Mass flow rate fluctuations through the swirler (continuous lines) and heat release fluctuations (dotted lines) for sectors 1, 3, 6 and 10. 


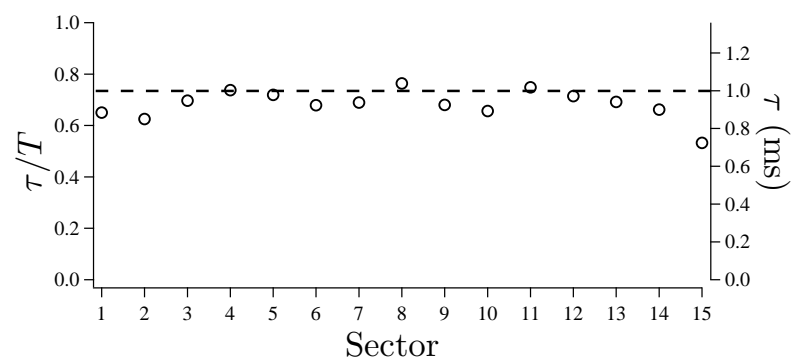

Figure 17: Time delay between mass flow rate and heat release fluctuations for all sectors (normalised by the first azimuthal mode period). The dashed line represents the value extracted from a pulsated single burner LES.

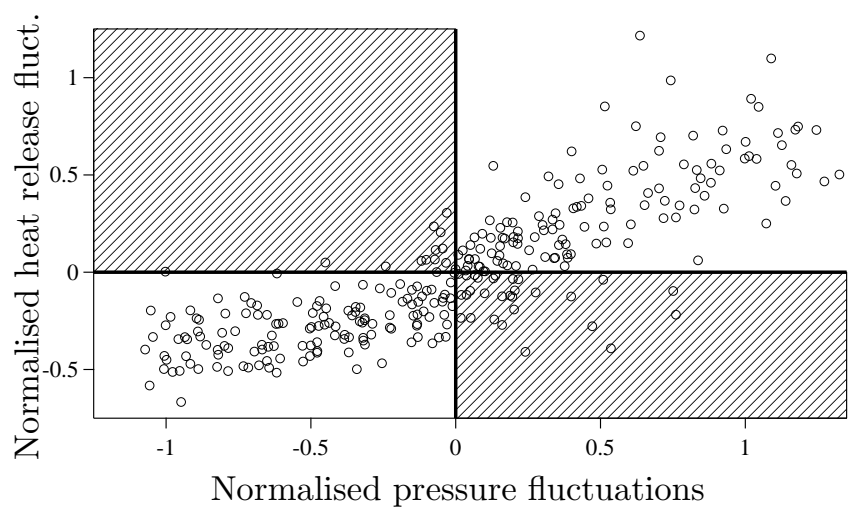

Figure 18: Heat release fluctuations versus pressure fluctuations at 20 phase instants for each sector. Striped zones indicate a non-fulfilled Rayleigh criterion $\left(p_{1} q_{1}>0\right)$.

considered by period. Furthermore, the domain is decomposed into 15 sectors, resulting in 300 fields, each one representing a given sector at a given phase instant. Spatial averaging is then performed to obtain a point representative of pressure and heat release fluctuations in each sector at each phase instant. Figure 18 shows the scatter plot that is obtained and reveals that, while most points seem correlated, a unique $\beta$ might not be sufficient to describe the flame. However, the general linear relationship between $p_{1}$ and $q_{1}$ postulated by [2] is verified. Furthermore, most points comply with the Rayleigh criterion $\left(p_{1} q_{1}>\right.$ 0), except those located in the striped zones of Fig. 18. 


\section{Stability prediction and control}

\subsection{Stability prediction}

The stability of the chamber can also be studied with the Helmholtz solver described in Section 3 and compared to LES predictions. Acoustic damping by the multi-perforated plates is included in the Helmholtz calculations through a homogeneous model $[68,69]$. Due to the low Mach number assumption of the solver, the domain is truncated upstream of the choked nozzle included in the LES (Fig. 19). Inlet and outlet boundary conditions are set to zero acoustic velocity $\left(u^{\prime}=0\right)^{2}$. The main inputs for the Helmholtz calculations, namely sound speed and flame transfer function (FTF), are extracted from a pulsated $3 \mathrm{D}$ reactive LES of a single sector, following the procedure described in [70]. Once several periods are simulated with the single sector LES, interaction index and $\tau$ fields are calculated via Direct Fourier Transform (DFT) at the pulsating frequency $f$ :

$$
n_{u}(\mathbf{x})=\left|\frac{\hat{\Omega}(\mathbf{x})}{\hat{u}(\mathbf{x})}\right| \quad \text { and } \quad \tau_{u}(\mathbf{x})=\frac{\arg \left(\frac{\hat{\Omega}(\mathbf{x})}{\hat{u}(\mathbf{x})}\right)}{2 \pi f}
$$

where $\hat{\Omega}$ and $\hat{u}$ are respectively the DFT of the heat release and velocity fluctuations.

The frequency of pulsation on the inlet is $750 \mathrm{~Hz}$, which is the frequency of the dominant azimuthal mode in this configuration (Fig. 6). From this single sector calculation, input fields for the Helmholtz solver are obtained (Fig. 19) and then duplicated 14 times around the turbine axis to build the full annular fields. Using a reference point located just upstream of the flame for each burner (Fig. 1), the flame transfer function is constructed through the local interaction index field, $n_{u}(\mathbf{x})$ and a delay. This delay can be the local delay $\tau_{u}(\mathbf{x})$ obtained by the LES. Another solution is to assume that the flame is

\footnotetext{
${ }^{2}$ Appendix Appendix A discusses the use of a complex impedance as inlet boundary condition.
} 

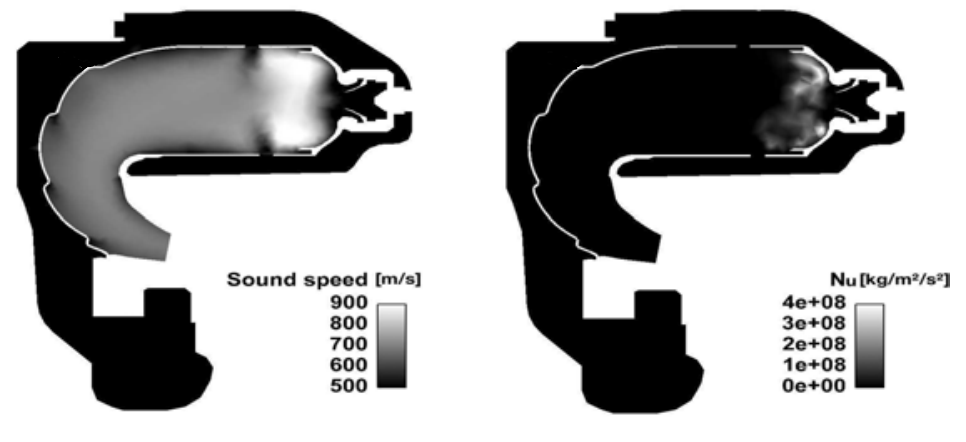

Figure 19: Sound speed (left) and local interaction index $n_{u}$ (right) fields on a planar cut of a single sector.

compact enough to have $\tau_{u}(\mathbf{x})=$ constant $=\tau$. Both methods will be used throughout this study, respectively called 'acoustic analysis with local delay' and 'with global delay'. An advantage of the latter method is that, by varying the global delay $\tau$, a stability chart can be built: the left part of Figure 20 displays the frequency of the first azimuthal mode, corresponding to the real part of the matching eigenfrequency found by the code. The imaginary part (right side of Fig. 20) measures the amplification of the mode. Positive amplification corresponds to an unstable behaviour, whereas negative amplification represents stable operation. This stability chart offers a qualitative description of the behaviour of the chamber when varying $\tau$ in the limit case of a compact flame. A discontinuity appears as the mode switches from stable to unstable around $\tau=\tau_{0}=0.610^{-3} \mathrm{~s}$, corresponding to $0.4 T_{a z i}$ where $T_{a z i}$ is the period of the first azimuthal mode. The frequency ranges from $570 \mathrm{~Hz}$ to $750 \mathrm{~Hz}$, depending on the delay.

If the flame is compact, its interaction index $n$ and global time delay $\tau$ can be evaluated from the fields of $n_{u}$ and $\tau_{u}$ :

$$
\iiint_{V} n_{u}(\mathbf{x}) e^{j \omega \tau_{u}(\mathbf{x})} d \mathcal{V}=n e^{j \omega \tau}
$$

The global time delay $\tau$ that would be equivalent to the field of local time delay $\tau_{u}$ extracted from the pulsated single sector LES is $\tau_{1}=1.0110^{-3} \mathrm{~s}$. This single sector delay value is also reported on Fig. 17 where it matches the delays 

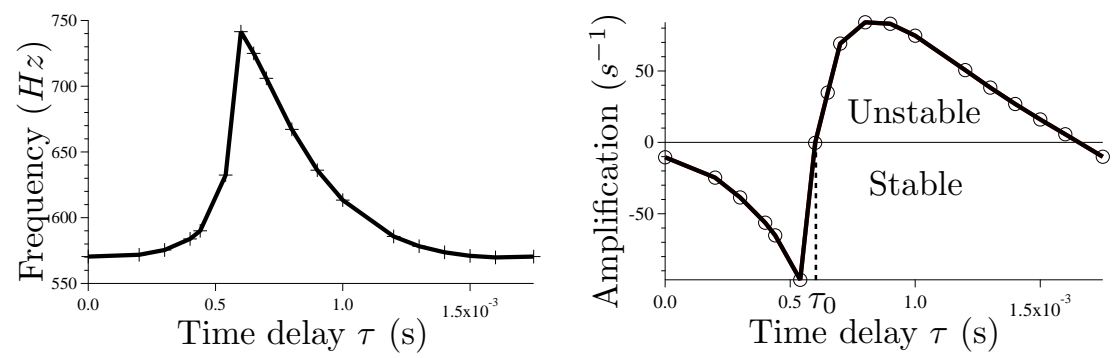

Figure 20: Stability chart: frequency (left) and amplification (right) versus global time delay for the first azimuthal mode.

\begin{tabular}{cccc}
\hline Mode & Frequency $(H z)$ & Amplification $\left(s^{-1}\right)$ & Structure \\
\hline $\mathbf{1}$ & 613 & 75 & Azimuthal \\
$\mathbf{2}$ & 621 & 67 & Azimuthal \\
$\mathbf{3}$ & 651 & -170 & Longitudinal \\
\hline
\end{tabular}

Table 4: Modes found by the Helmholtz solver for $\tau=\tau_{1}=1.0110^{-3} \mathrm{~s}$.

measured for all sectors in the full annular LES. The acoustic solver (Fig. 20) predicts this delay to lead to an unstable first azimuthal mode as observed also in the LES. Table 4 lists the modes found by the Helmholtz solver for $\tau=\tau_{1}$ that have a frequency smaller than $1000 \mathrm{~Hz}$. The first two modes are amplified azimuthal modes that have very close frequencies and amplifications. An observation of their structures (Fig. 21 and Fig. 22) indicates similar azimuthal structures with an almost constant modulus of pressure. Mode 1 has an anticlockwise phase whereas mode 2 has a clockwise phase, indicating turning modes. These two modes correspond to the "+" and "-" waves mentioned in Section 5 and can be combined as a single standing mode, as described in Section 5. The third mode is a longitudinal mode (no dependence of the modulus or the phase with the azimuthal angle $\theta$ as defined Fig. 2) that is predicted to be damped (Fig. 23). 


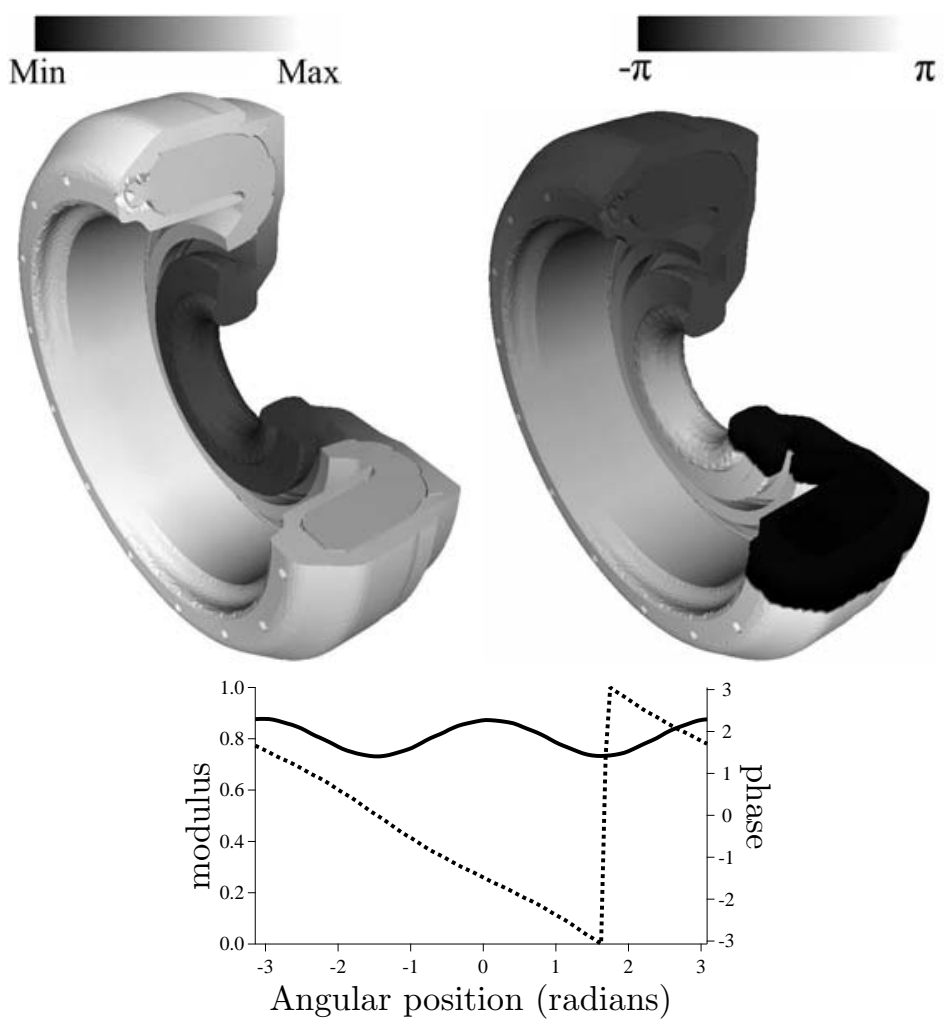

Figure 21: Mode structure obtained with the Helmholtz solver for the first azimuthal mode (613 Hz) shown in Table 4: pressure fluctuation modulus (left) and phase (right). Modulus (solid lines) and phase (dashed lines) in the chamber as function of the azimuthal angle. 


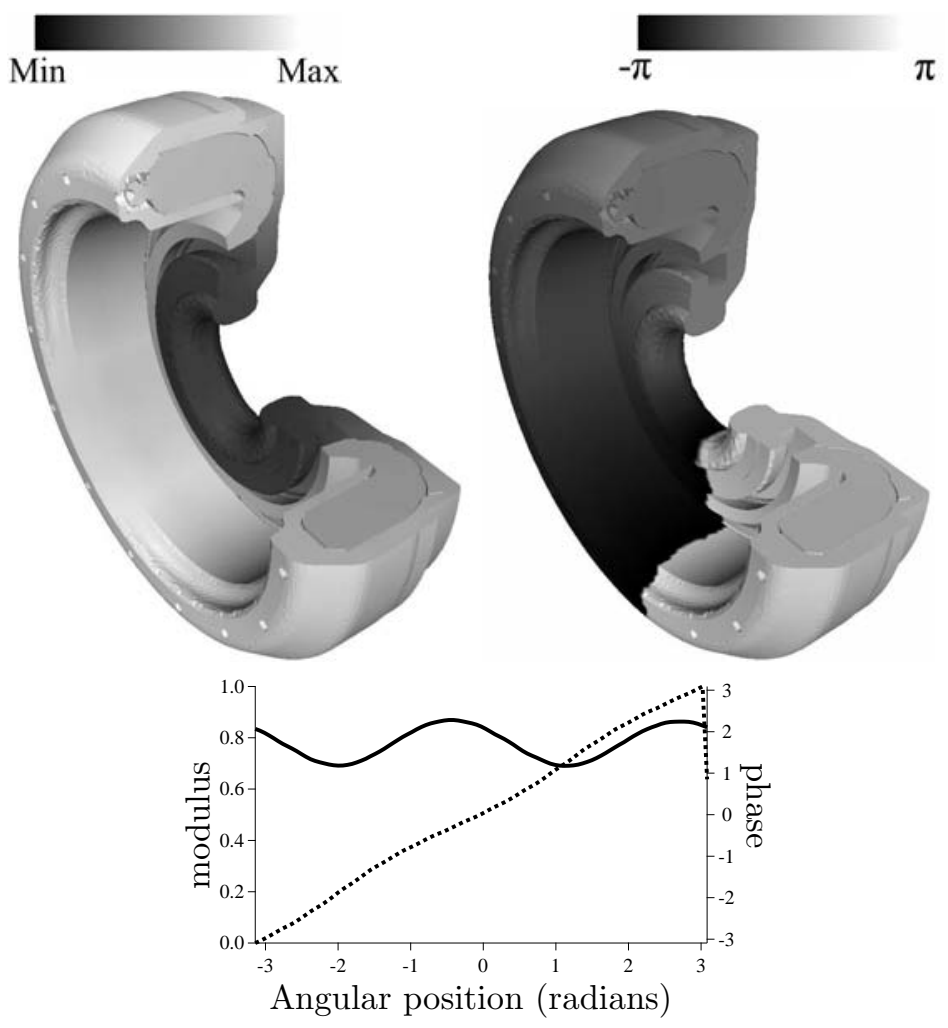

Figure 22: Mode structure obtained with the Helmholtz solver for the second azimuthal mode (621 Hz) shown in Table 4: pressure fluctuation modulus (left) and phase (right). Modulus (solid lines) and phase (dashed lines) in the chamber as function of the azimuthal angle. 

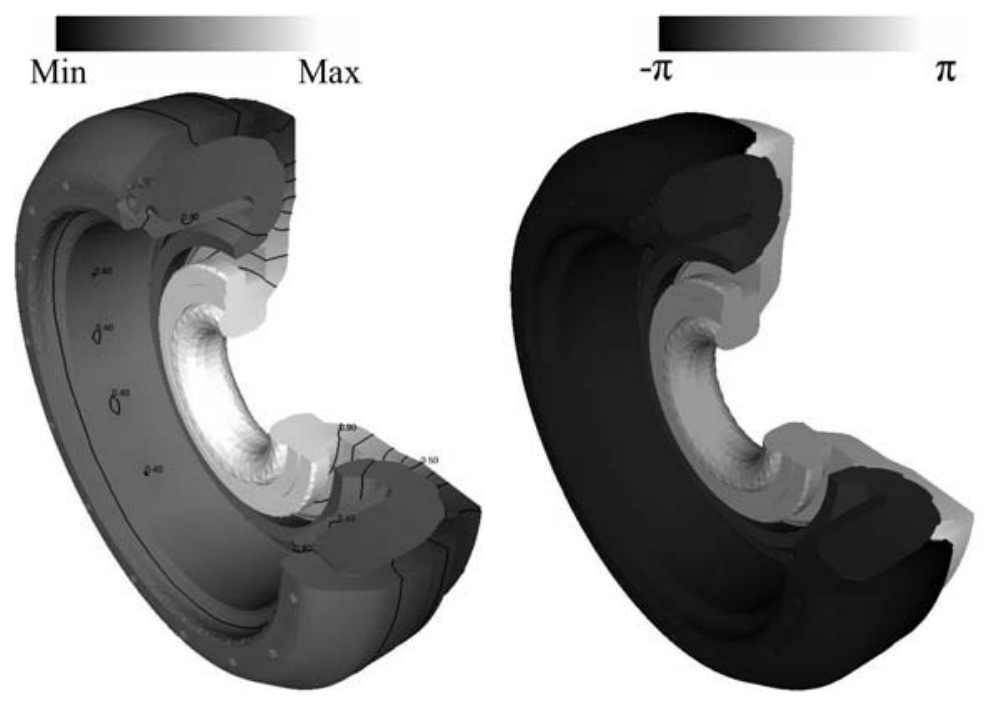

Figure 23: Mode structure for the longitudinal mode $(651 \mathrm{~Hz})$ shown in Table 4: pressure fluctuation modulus (left, with contours) and phase (right).

\subsection{Control of the instabilities by delay tuning}

Figure 20 shows that the delay $\tau$ of a single burner controls the stability of the full chamber and suggests that reducing $\tau$ would stabilise the azimuthal mode. This delay measures the time needed for a velocity perturbation at the reference point to induce a perturbation of heat release. If it is reduced below $\tau_{0}$, the critical delay for stability, Fig. 20 suggests that the mode should be damped. This section describes such a stabilisation exercise in which the delay of all burners will be decreased from $\tau_{1}$ to a value $\tau_{2}$, smaller than the critical value $\tau_{0}$. In a real combustor, this would require a change of the geometry or of the fuel characteristics. In the LES, this reduction of $\tau$ can be obtained simply by increasing the preexponential $A$ in the Arrhenius law (Eq. 4) and therefore the flame speed.

First, a pulsated single sector LES with a preexponential multiplied by four is carried out in order to extract the delay field $\tau_{u}(\mathbf{x})$. Using Eq. 15 one obtains a corresponding global delay $\tau=0.6910^{-3} \mathrm{~s}$, which is close to the stable/unstable transition found in Fig. 20. Helmholtz calculations are then made, this time 


\begin{tabular}{cccc}
\hline Chemistry & Equivalent global delay $\tau(s)$ & Frequency $(H z)$ & Amplification $\left(s^{-1}\right)$ \\
\hline Standard & $1.0110^{-3}$ & 633 & 32 \\
Fast & $0.6910^{-3}$ & 650 & -11 \\
\hline
\end{tabular}

Table 5: Frequency and amplification of the first azimuthal mode obtained by the Helmholtz solver for two different chemistries yielding different $n_{u}(\mathbf{x})$ and $\tau_{u}(\mathbf{x})$ fields.

taking into account the field of local time delay $\tau_{u}(\mathbf{x})$, for the standard and the fast chemistry (Table 5). The fast chemistry is predicted to be stable for the two azimuthal modes, contrary to the the standard one.

Full annular LES are then carried out with the fast chemistry. These calculations are initialised with fields coming from the standard chemistry full annular LES. Mean temperature fields for both the standard and the fast chemistry are displayed Fig. 24. There is no strong evidence of a difference in flame topology, although some burners in the fast chemistry simulation show a flame that is anchored somewhat upstream when compared to the standard chemistry.

While the flame positions with the standard and fast chemistries are very similar, an observation of the magnitude of pressure fluctuations reveals a totally different behavior between both chemistries (Fig. 25). The standard chemistry shows a strong level of fluctuations with two nodes and two antinodes, corresponding to the structure displayed on the left part of Fig. 9. On the other hand, the fast chemistry shows very weak pressure fluctuations. Most of the fluctuating activity is limited to the swirlers.

To quantify this reduction, merely plotting pressure signals at a local probe is not sufficient because of the low frequency modulation (Fig. 8) typical of Type 3 rotating modes (Table 1). However, since the mode structure is known, it is possible to use the indicator $C(t)$ proposed by [65] (Eq. 12). The time evolutions of this indicator for both standard and fast chemistry, considering 12 angular locations $\theta_{k}$, are displayed in Fig. 26. The fast chemistry simulation, started at $t=0.136 \mathrm{~ms}$, shows a drastic reduction of pressure fluctuations. After around 6 periods of the first azimuthal mode identified in the standard chemistry annular 


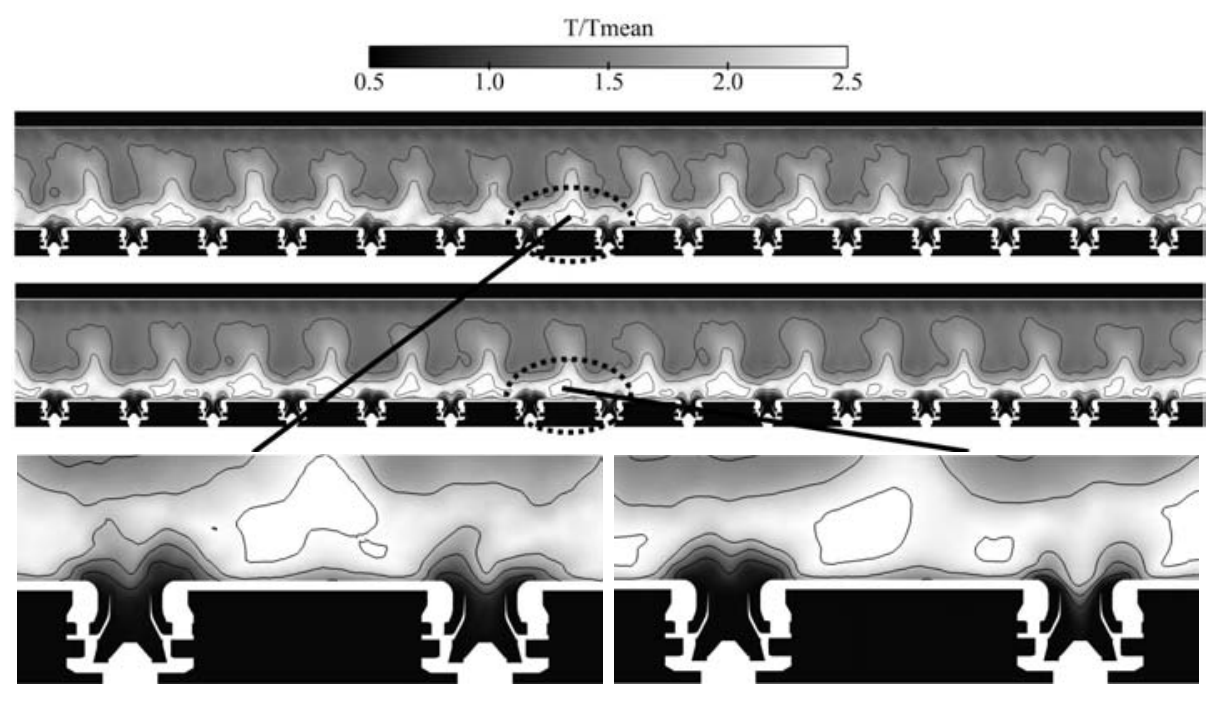

Figure 24: Mean temperature on a un-rolled surface of a cylinder passing through the $B_{i}$ probes. Top: standard chemistry showing all burners, middle: fast chemistry showing all burners. On the bottom left, a zoom on two burners is shown for the standard chemistry. On the bottom right, a zoom on two burners is shown for the fast chemistry.

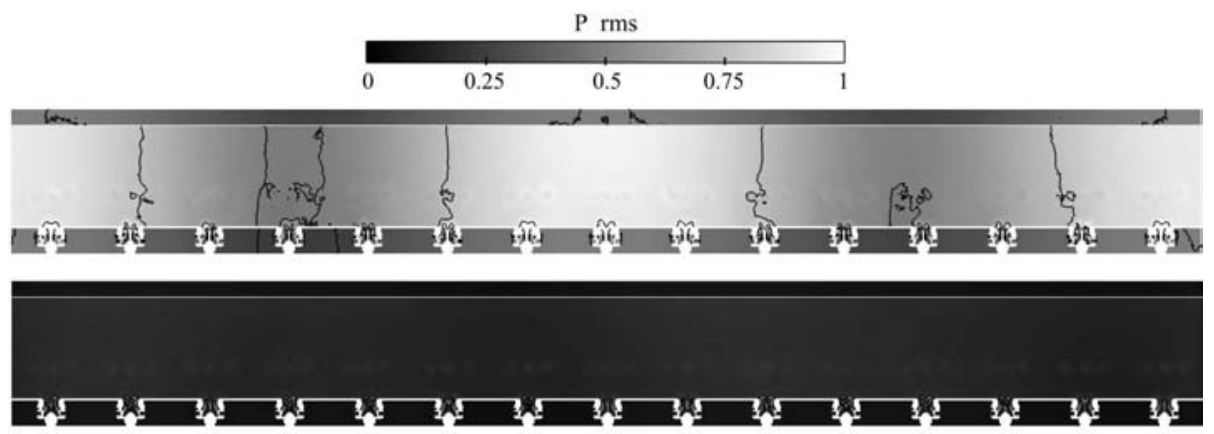

Figure 25: Magnitude of the pressure fluctuations on a un-rolled surface of a cylinder passing through the $B_{i}$ probes. Top: standard chemistry, bottom: fast chemistry. 


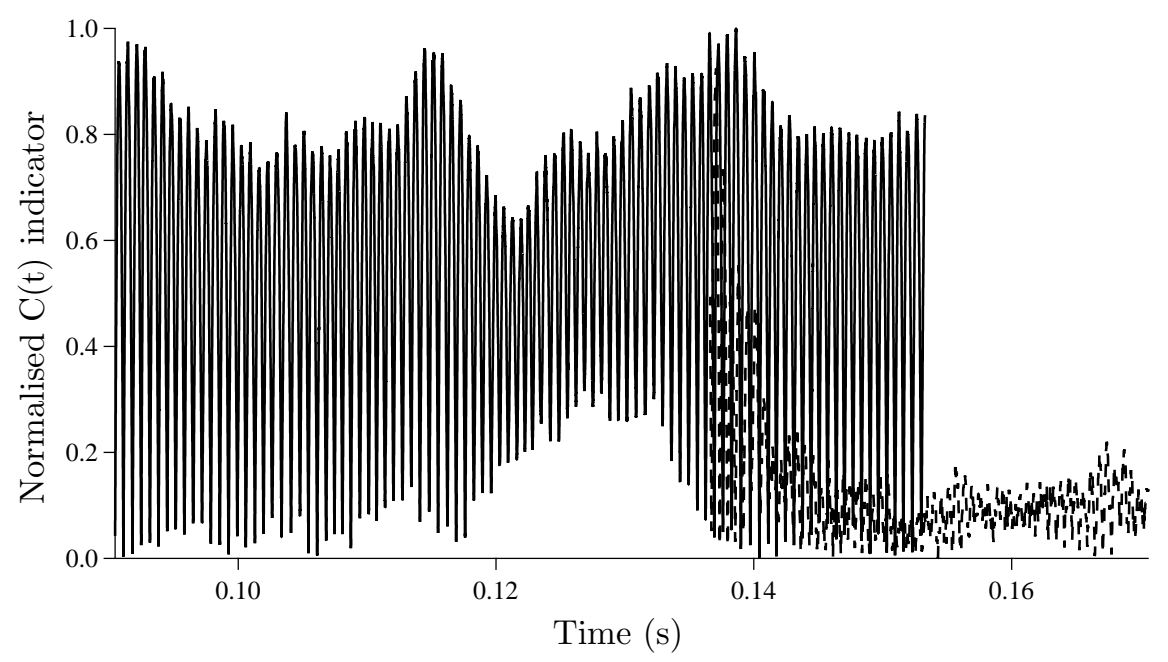

Figure 26: $C(t)$ indicator modulus, considering 12 probes evenly distributed, for the standard (continuous line) and the fast chemistry (dotted line).

LES, the value of $C(t)$ for the fast chemistry is damped by nearly an order of magnitude. Note also that the fluctuations of the standard chemistry case are harmonic at a frequency of $1500 \mathrm{~Hz}$, which is twice the frequency of the first azimuthal mode identified in Fig. 6 due to the construction of the indicator. The fast chemistry LES on the other hand presents a non-periodic indicator. Spectra of pressure fluctuations for both chemistries are shown in Fig. 27. A strong peak appears for the standard chemistry at $750 \mathrm{~Hz}$. On the other hand, the spectrum obtained with the fast chemistry confirms observations made with the $C(t)$ indicator and evidences a reduced level of quasi aperiodic fluctuations.

\section{Conclusion}

This paper describes a compressible Large Eddy simulation of the reacting flow in a full annular combustion chamber corresponding to a real helicopter engine. LES captures the azimuthal modes which develop in these chambers, both in frequency and amplitude range. More than 100 cycles of oscillation at the dominant first azimuthal mode frequency were computed and analysed: the observed modes are shown to be a combination of the two first azimuthal 


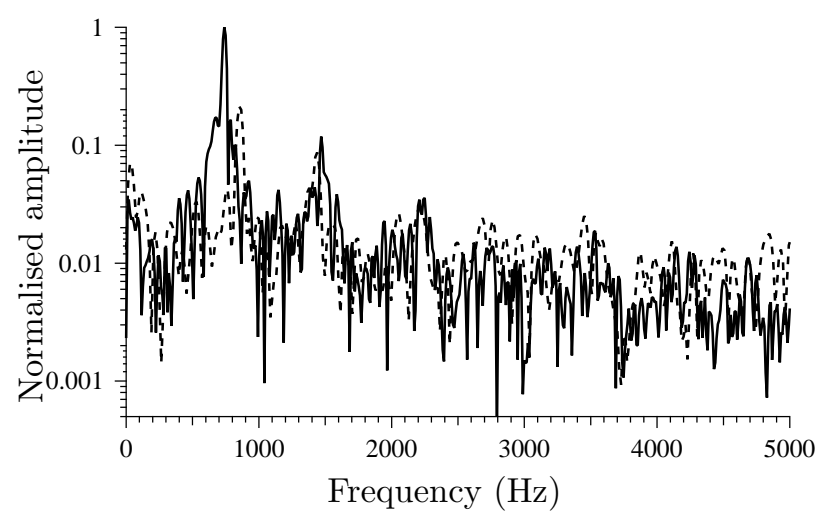

Figure 27: Pressure fluctuation spectra of the $B_{1}$ probe located in the combustion chamber for the standard (continuous line) and the fast chemistry (dotted line).

turning modes of the chamber with no dependance on the axial direction. The relative amplitudes of the azimuthal modes change with time so that the nature of the resulting pressure oscillations varies between purely standing and turning modes. However, the dominant mode is an almost purely standing mode. Moreover, a mean swirling convection velocity is created within the combustor so that the acoustic structure rotates slowly (at the mean swirl velocity). LES was also used to verify typical model assumptions, for example the linear dependance between pressure and heat release fluctuations postulated by [2]: the Rayleigh criterion was found to be mostly valid over the whole combustor.

In addition to the LES, an acoustic solver was used to compute stability maps. The results suggest that reducing the flame delay below a critical threshold should be sufficient to stabilise combustion. This was verified in the LES by artificially increasing the reaction kinetics, leading to a faster flame and a reduced flame delay. With this new chemistry, the LES stabilised to an oscillation free regime in 4 to 6 cycles, confirming the predictions of the acoustic solver.

Despite their costs, these results confirm that LES and three-dimensional acoustic analysis are powerful tools to investigate combustion instabilities even in complex geometries such as annular chambers. Embedding these steps at an early stage of conception will help creating intrinsically stable combustors. 


\section{Acknowledgments}

The authors wish to acknowledge fruitful discussions with Dr. B. Schuermans and Dr. N. Noiray. This research used resources of the Argonne Leadership Computing Facility at Argonne National Laboratory, which is supported by the Office of Science of the U.S. Department of Energy under contract DEAC02-06CH11357. The authors thank GENCI (Grand Equipement National de Calcul Intensif ) and CINES (Centre Informatique National de l'Enseignement Supérieur) for providing part of the computing power necessary for these simulations. The support of Turbomeca (Dr. C. Bérat and Dr. T. Lederlin) and ANRT/CIFRE is also acknowledged. This research is partly supported by the ANR under the project SIMTUR ANR-07-CIS7-008. 


\section{Appendix A. Acoustic boundary conditions uncertainties and their influence on Helmholtz calculations}

This section discusses the effects of acoustic boundary conditions on the results of the acoustic solver. These conditions are often difficult to specify: in a real engine, they are controlled by the compressor and the turbine. In the present LES, they are imposed by the numerical boundary conditions chosen at the inlet and outlet. Since the outlet is a choked section, where $u^{\prime}=0$ is a reasonable approximation in the low frequency limit [71, 72], this section focuses on the effects of the inlet condition. The frequency found by the Helmholtz solver for the first azimuthal eigenmode is $615 \mathrm{~Hz}$ when $u^{\prime}=0$ is used on all boundaries (inlet, outlet and walls). The discrepancy between the $750 \mathrm{~Hz}$ frequency measured in the full annular LES and the eigenfrequency found by the Helmholtz solver may come from uncertainties on the inlet boundary condition. Numerical experiments have been conducted without using a FTF (i.e. no modelling of the flame/acoustic interactions) and the frequency of the first azimuthal mode shifts from $615 \mathrm{~Hz}$ when considering null acoustic velocity on the air inlet (see Fig. 1) to $770 \mathrm{~Hz}$ when considering null acoustic pressure on the inlet. To evaluate the actual acoustic boundary condition in the full annular LES, the reflection coefficient $\mathcal{R}$ at the inlet is reconstructed from the full annular LES: $\mathcal{R}=\frac{w_{+}}{w_{-}}$, $w_{+}$being the acoustic wave that enters the domain and $w_{-}$the outgoing wave. Both waves are assumed to be planar waves propagating in the direction normal to the boundary condition. This reflection coefficient, displayed in Fig. A.28, is linked to the acoustic admittance $Y$ prescribed in the Helmholtz solver [30]:

$$
Y=\frac{\mathcal{R}-1}{\mathcal{R}+1}=\frac{\rho_{0} c_{0} \mathbf{u}^{\prime} \cdot \mathbf{n}_{\mathbf{B C}}}{p^{\prime}}
$$

In the low frequency regime, the inlet boundary condition used in the LES seems to behave as a null acoustic velocity condition, which can be rewritten as a Neumann condition for pressure, with the help of Eq. 6: $\nabla p^{\prime} \cdot \mathbf{n}_{\mathbf{B C}}=0$. However, in the frequency range of interest for the first azimuthal mode (600$800 \mathrm{~Hz}$ ), the inlet condition used in the LES seems to be less reflective. 

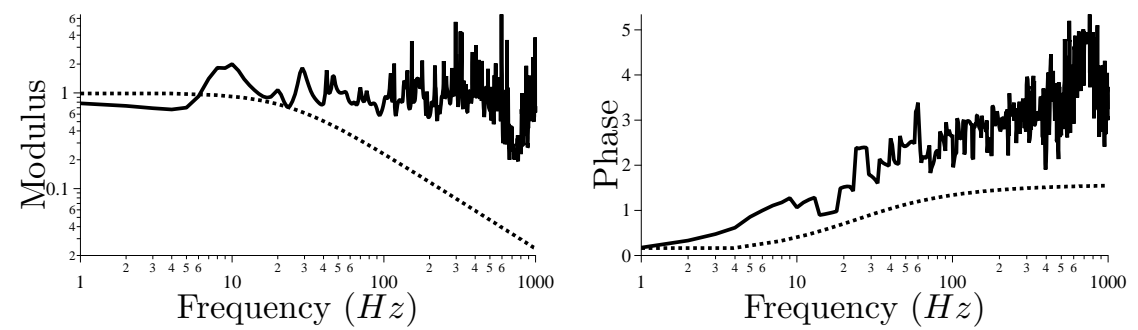

Figure A.28: Modulus and phase of the inlet reflection coefficient extracted from the full annular LES (continuous lines) and from the analytical model (dotted lines).

In the LES, the inlet condition is imposed using the NSCBC method [10]: the amplitude $\mathcal{L}_{5}$ of the incoming acoustic wave is proportional to $u-u_{t}$, where $u$ is the instantaneous local velocity and $u_{t}$ the target local velocity.

This ensures an almost non-reflecting boundary condition while avoiding drifts in the mean imposed flow [10,73]. Based on this description, one can derive the reflection coefficient of the inlet used in the LES in a similar fashion to the derivation proposed for an outlet in [73]:

$$
\mathcal{R}=\frac{\mathcal{L}_{5}}{\mathcal{L}_{1}}=\frac{1}{1-\frac{2 j \rho c \omega}{K}}
$$

Modulus and phase of the inlet reflection coefficient are then respectively:

$$
\begin{array}{r}
|\mathcal{R}|=\frac{1}{\sqrt{1+\left(\frac{2 \rho c \omega}{K}\right)^{2}}} \\
\Phi=\arg (\mathcal{R})=\arctan \left(\frac{2 \rho c \omega}{K}\right)
\end{array}
$$

These are plotted in Fig. A.28 in dotted lines. Both the modulus and phase of the model agree well with the LES in the low frequency domain, where the boundary condition behaves acoustically as a totally reflective wall. The model of Eqs. A.3 and A.4 then becomes less reflective for a much lower frequency than the reconstructed reflection coefficient, while its phase shifts from 0 to $\pi / 2$. An increase is also seen for the phase of the LES reconstructed coefficient, although the phase reaches $\pi$ for high frequencies, and even $3 \pi / 2$ around $750 \mathrm{~Hz}$. Results for the Helmholtz solver simulations obtained for the different boundary 


\begin{tabular}{ccc}
\hline Boundary condition & Frequency $(H z)$ & Amplification $\left(s^{-1}\right)$ \\
\hline$u^{\prime}=0$ & 613 & 75 \\
$Y_{\text {LES }}$ & 630 & 23 \\
$Y_{\text {model }}$ & 611 & 29 \\
\hline
\end{tabular}

Table A.6: Frequency and amplification obtained by the Helmholtz solver for three different boundary conditions: $u^{\prime}=0$, the admittance derived from the full annular LES reflection coefficient $\left(Y_{L E S}\right)$ and the admittance obtained for the modelled NSCBC inlet used in the LES $\left(Y_{\text {model }}\right)$. The global time delay $\tau$ is $1.010^{-3} \mathrm{~s}$.

conditions are summarised in Table A.6. Frequencies are found to be fairly close. The mode is found to be unstable for all boundary conditions, with positive amplifications. However, while both boundary conditions using admittance are predicted to have similar amplifications, the $u^{\prime}=0$ condition is found to be more amplified.

\section{Appendix B. An indicator for azimuthal mode type}

This appendix shows how $\mathrm{N}$ pressure probes in an annular chamber can be used to identify azimuthal modes. An indicator $C(t)$ is defined as:

$$
C(t)=\frac{1}{N} \sum_{k=1}^{N} p_{1}\left(\theta_{k}, t\right) e^{j \theta_{k}}
$$

where $\mathrm{N}$ evenly distributed pressure signals around the azimuthal direction are taken into account (Probes are spaced by $2 \pi / N$ radians). The real valued pressure signal at probe $k$ can be written as:

$$
p_{1}\left(\theta_{k}, t\right)=A \cos \left(\theta_{k}-\phi(t)\right) \text { for a turning mode }
$$

and

$$
p_{1}\left(\theta_{k}, t\right)=2 A \cos \left(\theta_{k}\right) \cos (\phi(t)) \text { for a standing mode }
$$

For a right turning mode: $\phi(t)=\omega t$ so that

$$
C(t)=\frac{A}{2} e^{j \omega t}
$$




\begin{tabular}{lcccl}
\hline & $\mathrm{C}(\mathrm{t})$ & $|C(t)|$ & Phase of $\mathrm{C}(\mathrm{t})$ & \\
\hline Type 1 or 3 & $A \cos (\omega t)$ & $A|\cos (\omega t)|$ & constant & Standing \\
Type 2 & Right turning: $\frac{A}{2} e^{j \omega t}$ & $\frac{A}{2}$ & Right turning: $\omega t$ & Turning \\
& Left turning: $\frac{A}{2} e^{-j \omega t}$ & & Left turning: $-\omega t$ & \\
\hline
\end{tabular}

Table B.7: Classification of modes and corresponding $\mathrm{C}(\mathrm{t})$ indicator.

For a left turning mode: $\phi(t)=-\omega t$ and

$$
C(t)=\frac{A}{2} e^{-j \omega t}
$$

If the mode is standing, $\phi(t)=-\omega t$ and

$$
C(t)=A \cos (\omega t)
$$

Table Appendix B summarises how $C(t)$ can be used to identify the nature of the mode: standing modes (Type 1 or 3 ) are characterised by a modulus of $C(t)$ that oscillates with a pulsation $2 \omega$ and a constant phase. Turning modes (Type 2) will lead to a constant modulus of $C(t)$ and a phase that linearly evolves with time.

\section{References}

[1] W. Krebs, P. Flohr, B. Prade, S. Hoffmann, Thermoacoustic stability chart for high intense gas turbine combustion systems, Combust. Sci. Tech. 174 (2002) 99-128.

[2] N. Noiray, M. Bothien, B. Schuermans, Investigation of azimuthal staging concepts in annular gas turbines, Combustion Theory and Modelling 15 (4).

[3] B. Schuermans, V. Bellucci, C. Paschereit, Thermoacoustic modeling and control of multiburner combustion systems, in: International Gas Turbine and Aeroengine Congress \& Exposition, ASME Paper, Atlanta, USA, 2003. 
[4] T. Lieuwen, V. Yang, Combustion Instabilities in Gas Turbine Engines. Operational Experience, Fundamental Mechanisms and Modeling, Prog. in Astronautics and Aeronautics AIAA, Vol. 210, 2005.

[5] L. Rayleigh, The explanation of certain acoustic phenomena, Nature July 18 (1878) 319-321.

[6] L. Crocco, Research on combustion instability in liquid propellant rockets., in: 12th Symp. (Int.) on Combustion, The Combustion Institute, Pittsburgh, 1969, pp. 85-99.

[7] N. Noiray, D. Durox, T. Schuller, S. Candel, A unified framework for nonlinear combustion instability analysis based on the flame describing function a unified framework for nonlinear combustion instability analysis based on the flame describing function, J. Fluid Mech. 615 (2008) 139-167.

[8] T. Lieuwen, Modeling premixed combustion-acoustic wave interactions: A review, J. Prop. Power 19 (5) (2003) 765-781.

[9] A. P. Dowling, The calculation of thermoacoustic oscillations, J. Sound Vib. 180 (4) (1995) 557-581.

[10] T. Poinsot, D. Veynante, Theoretical and Numerical Combustion, R.T. Edwards, 2nd edition, 2005.

[11] D. G. Crighton, A. P. Dowling, J. E. F. Williams, M. Heckl, F. Leppington, Modern methods in analytical acoustics, no. XVII in Lecture Notes, Springer Verlag, New-York, 1992.

[12] W. Polifke, A. Poncet, C. O. Paschereit, K. Doebbeling, Reconstruction of acoustic transfer matrices by instationnary computational fluid dynamics, J. Sound Vib. 245 (3) (2001) 483-510.

[13] A. L. Birbaud, D. Durox, S. Ducruix, S. Candel, Dynamics of confined premixed flames submitted to upstream acoustic modulations, Proc. Combust. Inst. 31 (2007) 1257-1265. 
[14] K. Truffin, T. Poinsot, Comparison and extension of methods for acoustic identification of burners, Combust. Flame 142 (4) (2005) 388-400.

[15] F. Boudy, D. Durox, T. Schuller, S. Candel, Nonlinear mode triggering in a multiple flame combustor, Proc. Combust. Inst. 33 (2011) 1121-1128.

[16] F. Nicoud, T. Poinsot, Thermoacoustic instabilities: should the rayleigh criterion be extended to include entropy changes ?, Combust. Flame 142 (2005) 153-159.

[17] G. Walz, W. Krebs, S. Hoffmann, H. Judith, Detailed analysis of the acoustic mode shapes of an annular combustion chamber, J. Engineering for Gas Turbines and Power 124 (1) (2002) 3-9.

[18] S. R. Stow, A. P. Dowling, Thermoacoustic oscillations in an annular combustor, in: International Gas Turbine and Aeroengine Congress \& Exposition, ASME Paper, New Orleans, Louisiana, 2001.

[19] A. S. Morgans, S. R. Stow, Model-based control of combustion instabilities in annular combustors, Combust. Flame 150 (4) (2007) 380-399.

[20] S. Evesque, W. Polifke, C. Pankiewitz, Spinning and azimuthally standing acoustic modes in annular combustors, in: 9th AIAA/CEAS Aeroacoustics Conference, South Carolina, USA, 2003.

[21] M. Lohrmann, H. Buechner, N. Zarzalis, Flame transfer function characteristics of swirled flames for gas turbine applications, in: ASME Turbo expo, Atlanta, USA, 2003.

[22] G. Staffelbach, L. Gicquel, G. Boudier, T. Poinsot, Large eddy simulation of self-excited azimuthal modes in annular combustors, Proc. Combust. Inst. 32 (2009) 2909-2916.

[23] T. Poinsot, A. Trouvé, D. Veynante, S. Candel, E. Esposito, Vortex driven acoustically coupled combustion instabilities, J. Fluid Mech. 177 (1987) 265-292. 
[24] T. Lieuwen, B. T. Zinn, The role of equivalence ratio oscillations in driving combustion instabilities in low nox gas turbines, Proc. Combust. Inst. 27 (1998) 1809-1816.

[25] A. Giauque, L. Selle, T. Poinsot, H. Buechner, P. Kaufmann, W. Krebs, System identification of a large-scale swirled partially premixed combustor using LES and measurements, J. Turb. 6 (21) (2005) 1-20.

[26] J. O'Connor, S. Natarajan, M. Malanoski, T. Lieuwen, Disturbance field characteristics of a transversely excited swirl flame, in: ASME Turbo Expo 2010, Glasgow, U.K., 2010.

[27] K. Kedia, H. Altay, A. Ghoniem, Impact of flame-wall interaction on premixed flame dynamics and transfer function characteristics, Proc. Combust. Inst. 33 (2011) 1113-1120.

[28] S. Candel, D. Durox, S. Ducruix, A. Birbaud, N. Noiray, T. Schuller, Flame dynamics and combustion noise : progress and challenges., Int. J. Acoust. 8 (2009) $1-56$.

[29] C. Pankiewitz, T. Sattelmayer, Time domain simulation of combustion instabilities in annular combustors, ASME Journal of Engineering for Gas Turbines and Power 125 (3) (2003) 677-685.

[30] F. Nicoud, L. Benoit, C. Sensiau, T. Poinsot, Acoustic modes in combustors with complex impedances and multidimensional active flames, AIAA Journal 45 (2007) 426-441.

[31] P. Wolf, G. Staffelbach, R. Balakrishnan, A. Roux, T. Poinsot, Azimuthal instabilities in annular combustion chambers, in: Proc. of the Summer Program, Center for Turbulence Research, NASA AMES, Stanford University, USA, 2010.

[32] B. Schuermans, C. Paschereit, P. Monkiewitz, Non-linear combustion instabilities in annular gas-turbine combustors, in: 44th AIAA Aerospace Sciences Meeeting and Exhibit, Reno, Nevada, 2006. 
[33] C. Sensiau, Simulations numériques des instabilités thermoacoustiques dans les chambres de combustion aéronautiques - th/cfd/08/127, Ph.D. thesis, Université de Montpellier II, - Institut de Mathématiques et de Modélisation de Montpellier, France (2008).

[34] N. Noiray, M. Bothien, B. Schuermans, Analytical and numerical analysis of staging concepts in annular gas turbines, in: N3L - International Summer School and Workshop on Non-normal and nonlinear effects in aero and thermoacoustics, T.U.M Munich, Germany, 2010.

[35] J. P. Moeck, M. Paul, C. O. Paschereit, Thermoacoustic instabilities in an annular rijke tube, in: ASME Turbo Expo 2010, Glasgow, U.K., 2010.

[36] P. Moin, S. V. Apte, Large-eddy simulation of realistic gas turbine combustors, Am. Inst. Aeronaut. Astronaut. J. 44 (4) (2006) 698-708.

[37] K. Mahesh, G. Constantinescu, S. Apte, G. Iaccarino, F. Ham, P. Moin, Large eddy simulation of reacting turbulent flows in complex geometries, ASME J. Appl. Mech. 73 (2006) 374-381.

[38] P. Schmitt, T. Poinsot, B. Schuermans, K. P. Geigle, Large-eddy simulation and experimental study of heat transfer, nitric oxide emissions and combustion instability in a swirled turbulent high-pressure burner, J. Fluid Mech. 570 (2007) 17-46. doi:10.1017/S0022112006003156.

[39] L. Selle, G. Lartigue, T. Poinsot, R. Koch, K.-U. Schildmacher, W. Krebs, B. Prade, P. Kaufmann, D. Veynante, Compressible large-eddy simulation of turbulent combustion in complex geometry on unstructured meshes, Combust. Flame 137 (4) (2004) 489-505.

[40] P. Wolf, G. Staffelbach, A. Roux, L. Gicquel, T. Poinsot, V. Moureau, Massively parallel LES of azimuthal thermo-acoustic instabilities in annular gas turbines, C. R. Acad. Sci. Mécanique 337 (6-7) (2009) 385-394.

[41] C. Fureby, LES of a multi-burner annular gas turbine combustor, Flow, Turbulence and Combustion 84 (3) (2010) 543-564. 
[42] W. Lang, T. Poinsot, S. Candel, Active control of combustion instability, Combust. Flame 70 (1987) 281-289.

[43] G. Bloxsidge, A. Dowling, N. Hooper, P. Langhorne, Active control of reheat buzz, AIAA Journal 26 (1988) 783-790.

[44] G. Billoud, M. Galland, C. Huynh, S. Candel, Adaptive active control of combustion instabilities, Combust. Sci. Tech. 81 (1992) 257-283.

[45] J. Seume, N. Vortmeyer, W. Krause, J. Hermann, C. Hantschk, P. Zangle, S. Gleis, D. Vortmeyer, A. Orthmann, Application of active combustion instability control to a heavy gas turbine, J. Engineering for Gas Turbines and Power 120 (1998) 721-726.

[46] C. Martin, L. Benoit, Y. Sommerer, F. Nicoud, T. Poinsot, LES and acoustic analysis of combustion instability in a staged turbulent swirled combustor, AIAA Journal 44 (4) (2006) 741-750.

[47] V. Moureau, G. Lartigue, Y. Sommerer, C. Angelberger, O. Colin, T. Poinsot, Numerical methods for unsteady compressible multi-component reacting flows on fixed and moving grids, J. Comput. Phys. 202 (2) (2005) 710-736. doi:10.1016/j.jcp.2004.08.003.

[48] N. Gourdain, L. Gicquel, M. Montagnac, O. Vermorel, M. Gazaix, G. Staffelbach, M. Garcia, J. Boussuge, T. Poinsot, High performance parallel computing of flows in complex geometries: I. methods, Comput. Sci. Disc. 2 (2009) 015003.

[49] O. Colin, M. Rudgyard, Development of high-order taylor-galerkin schemes for unsteady calculations, J. Comput. Phys. 162 (2) (2000) 338-371.

[50] J. Smagorinsky, General circulation experiments with the primitive equations: 1. the basic experiment., Mon. Weather Rev. 91 (1963) 99-164.

[51] T. Poinsot, S. Lele, Boundary conditions for direct simulations of compressible viscous flows, J. Comput. Phys. 101 (1) (1992) 104-129. doi:10.1016/0021-9991(92)90046-2. 
[52] S. Mendez, F. Nicoud, Adiabatic homogeneous model for flow around a multiperforated plate, Am. Inst. Aeronaut. Astronaut. J. 46 (10) (2008) 2623-2633.

[53] S. Mendez, F. Nicoud, Large-eddy simulation of a bi-periodic turbulent flow with effusion, J. Fluid Mech. 598 (2008) 27-65.

[54] S. C. Li, B. Varatharajan, F. A. Williams, Chemistry of JP-10 ignition, Am. Inst. Aeronaut. Astronaut. J. 39 (12) (2001) 2351-2356.

[55] G. Boudier, L. Y. M. Gicquel, T. Poinsot, D. Bissières, C. Bérat, Effect of mesh resolution on large eddy simulation of reacting flows in complex geometry combustors, Combust. Flame 155 (1-2) (2008) 196-214.

[56] J.-P. Légier, Simulations numériques des instabilités de combustion dans les foyers aéronautiques, Phd thesis, INP Toulouse (2001).

[57] B. Franzelli, E. Riber, M. Sanjosé, T. Poinsot, A two-step chemical scheme for Large-Eddy Simulation of kerosene-air flames, Combust. Flame 157 (7) (2010) 1364-1373.

[58] O. Colin, F. Ducros, D. Veynante, T. Poinsot, A thickened flame model for large eddy simulations of turbulent premixed combustion, Phys. Fluids 12 (7) (2000) 1843-1863.

[59] J.-P. Légier, T. Poinsot, D. Veynante, Dynamically thickened flame LES model for premixed and non-premixed turbulent combustion, in: Proc. of the Summer Program, Center for Turbulence Research, NASA AMES, Stanford University, USA, 2000, pp. 157-168.

[60] L. Crocco, Aspects of combustion instability in liquid propellant rocket motors. part I., J. American Rocket Society 21 (1951) 163-178.

[61] C. Sensiau, F. Nicoud, T. Poinsot, A tool to study azimuthal and spinning modes in annular combustors, Int. Journal Aeroacoustics 8 (1) (2009) 57 68. 
[62] T. Poinsot, Analyse des instabilités de combustion de foyers turbulents prémélangés, Thèse d'etat, Université d'Orsay (1987).

[63] F. Hlawatsch, F. Auger, Time-frequency analysis: concepts and methods, Wiley-ISTE, 2008.

[64] P. Wolf, R. Balakrishnan, G. Staffelbach, L. Gicquel, T. Poinsot, Using LES to study reacting flows and instabilities in annular combustion chambers, Flow, Turb. and Combustion (accepted).

[65] B. Schuermans, An indicator for azimuthal mode type, private communication (07 2011).

[66] J. Hermann, A. Orthmann, S. Hoffmann, P. Berenbrink, Combination of active instability control and passive measures to prevent combustion instabilities in a $260 \mathrm{mw}$ heavy duty gas turbine, in: RTO AVT Symposium, Braunschweig, 2000.

[67] S. Evesque, W. Polifke, Low-order acoustic modelling for annular combustors: Validation and inclusion of modal coupling, in: International Gas Turbine and Aeroengine Congress \& Exposition, ASME Paper, Amsterdam, Netherlands, 2002.

[68] M. S. Howe, On the theory of unsteady high reynolds number flow through a circular aperture, Proc. R. Soc. Lond. A, Mathematical and Physical Sciences 366 (1725) (1979) 205-223.

[69] E. Gullaud, S. Mendez, C. Sensiau, F. Nicoud, T. Poinsot, Effect of multiperforated plates on the acoustic modes in combustors, C. R. Acad. Sci. Mécanique 337 (6-7) (2009) 406-414.

[70] A. Kaufmann, F. Nicoud, T. Poinsot, Flow forcing techniques for numerical simulation of combustion instabilities, Combust. Flame 131 (2002) 371385. 
[71] F. E. Marble, S. Candel, Acoustic disturbances from gas nonuniformities convected through a nozzle, J. Sound Vib. 55 (1977) 225-243.

[72] S. R. Stow, A. P. Dowling, T. P. Hynes, Reflection of circumferential modes in a choked nozzle, Journal of Fluid Mechanics 467 (2002) 215-239. doi:10.1017/S0022112002001428.

[73] L. Selle, F. Nicoud, T. Poinsot, The actual impedance of non-reflecting boundary conditions: implications for the computation of resonators, AIAA Journal 42 (5) (2004) 958-964. 